\title{
Continuum solutions for tunnel-building interaction and a modified framework for deformation prediction
}

\author{
A. FRANZA*, S. RITTER $\dagger$ and M. J. DEJONG $\ddagger$
}

\begin{abstract}
In this paper, the response of buildings to tunnelling-induced ground movements is studied with elastic and elastoplastic continuum solutions that consider the structure as an equivalent simple beam. A comparison is made between these simple solutions and centrifuge test data to provide insights into flexural and axial building deformations of low-rise bearing wall structures on strip foundations; the influence of wall openings and the foundation scheme on the equivalent beam bending stiffness is also addressed. Subsequently, the effects of structural continuity across greenfield sagging and hogging regions on tunnel-structure interaction are investigated. Finally, the continuum solutions are used to propose a modification factor formulation that accounts for the change in settlement trough shape (compared to the greenfield) due to soil-structure interaction. This formulation, for example, accounts for the change in transverse length of the hogging and sagging regions of a building due to soilstructure interaction, eliminating the need to divide the building at the greenfield inflection points when calculating modification factors. The proposed formulation, which is compared with numerical, experimental and field data from previous research, is shown to better predict flexural building deformations.
\end{abstract}

KEYWORDS: Tunnels \& tunnelling, settlement, soil/structure interaction, elasticity, centrifuge modelling

\section{INTRODUCTION}

The development of urban areas often requires the excavation of new tunnels; this process can affect surface buildings and infrastructure. Tunnelling-induced structure deformations depend on ground movements, structure stiffness, load condition, and structural configuration (both foundation and superstructure). In particular, the role of structure characteristics in tunnel-structure interaction (TSI) has been primarily investigated with numerical modelling and field data (Bilotta et al., 2017; Dimmock \& Mair, 2008; Farrell et al., 2014; Franzius et al., 2006; Giardina et al., 2015; Fargnoli et al., 2015; Losacco et al., 2014; Mair, 2013; Pickhaver et al., 2010), although centrifuge tests of TSI have also been performed (Farrell et al., 2014; Franza \& Marshall, 2018; Ritter et al., 2017a,b; Taylor \& Grant, 1998; Taylor \& Yip, 2001).

This paper aims to illustrate, through comparison with centrifuge data, the efficiency of simple continuum solutions in predicting the tunnelling-induced deformations of low-rise bearing wall structures on strip foundations. Then, using these continuum solutions, the influence of structure continuity across sagging and hogging greenfield settlement regions on TSI is investigated. Finally, a modified framework for the estimation of flexural modification factors is proposed.

\section{BACKGROUND}

Greenfield tunnelling in soft soils

Tunnelling induces both vertical $\left(u_{z}\right)$ and horizontal $\left(u_{x}\right)$ ground movements at the surface. Greenfield tunnelling-induced ground movements are often assessed, as shown in Figure 1, with empirical methods based on Gaussian curves and the tunnel volume loss parameter $\left(V_{l, t}\right)$, which is the ground loss at the tunnel periphery per unit length of tunnel normalised by the tunnel area.

\footnotetext{
Manuscript received. . .

* ETSI Caminos, Canales y Puertos, Technical University of Madrid, Madrid, Spain. Formerly, Department of Engineering, University of Cambridge, Cambridge, UK.

$\dagger$ Department of Engineering, University of Cambridge, Cambridge, UK.

$\ddagger$ Department of Civil and Environmental Engineering, University of California, Berkeley, USA.
} 


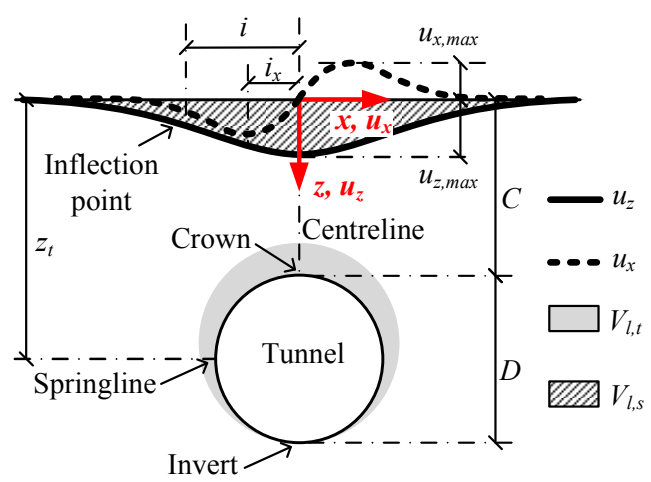

Fig. 1. Surface ground movements and volume losses.

In clays, surface settlement troughs are predicted with the use of standard Gaussian curves displayed in Equation (1) (Peck, 1969; Mair et al., 1993).

$$
u_{z}=u_{z, \max } \exp \left(-\frac{x^{2}}{2 i^{2}}\right)
$$

where $x$ is the horizontal spatial coordinate, $u_{z, \max }$ is the maximum settlement, $i$ is the horizontal distance of the settlement trough inflection point to the tunnel centreline, and $z_{t}$ is the tunnel axis depth.

In sandy soils, modified Gaussian curves (Equation (2)) better replicate settlement troughs (Vorster et al., 2005); trough width increases with $C / D$ and decreases with $V_{l, t}$, whereas the dependence on the relative soil density varies with $C / D$ (Franza et al., 2018; Marshall et al., 2012; Sugiyama et al., 1999). For sandy soils, a good fit to surface $u_{x}$ is achievable with Equation (3) (Farrell, 2010).

$$
\begin{gathered}
u_{z}=\frac{u_{z, \max } \cdot n}{(n-1)+\exp \left[a(x / i)^{2}\right]} ; n=e^{a} \frac{2 a-1}{2 a+1}+1 \\
u_{x}=u_{x, \max } \frac{1.65 x}{i_{x}} \exp \left[-\frac{x^{2}}{2 i_{x}^{2}}\right]
\end{gathered}
$$

where $n$ is the shape factor (if $n=1$, Equation (2) is the standard Gaussian curve), $a$ is the parameter to ensure that $i$ remains the distance to the inflection point, and $i_{x}$ is the horizontal offset of the maximum horizontal displacement, $u_{x, \text { max }}$.

\section{Continuum solutions}

The two-stage solutions proposed by Franza \& DeJong (2018), incorporated into a computer program 'ASRE', were adopted in this paper. As shown in Figure 2, the surface structure is simulated by an Euler-Bernoulli elastic beam with no shear deformability and its axis located at the ground level. Structural loads are simulated by a distributed vertical load at the beam axis. The structure is connected to vertical and horizontal coupled springs that model the elastic homogeneous half-space continuum, the soil. As basic assumptions, the tunnel and structure presence does not influence, respectively, the response of the continuum and the tunnelling process. The cross-sectional properties and Young's modulus of the equivalent beam are chosen to represent the axial stiffness and bending stiffness of both the foundation and the superstructure combined. To allow for the separation and relative soil-structure sliding, perfectly plastic sliders are located between the soil springs and the structure. The tunnel-structure interaction (TSI) is solved, after applying vertical loads to the structure, by imposing surface greenfield movements to the springs in incremental steps.

The finite element method (FEM) is used to solve the TSI problem numerically, which is described by the following equilibrium equations

$$
\begin{aligned}
& \left(\mathbf{S}+\mathbf{K}^{*}\right) \mathbf{u}=\mathbf{p}+\mathbf{K}^{*}\left(\mathbf{u}^{c a t}+\mathbf{\Lambda}^{*}\langle(\mathbf{p}-\mathbf{S u})\rangle+\mathbf{u}^{s l}\right) \\
& \langle(\mathbf{p}-\mathbf{S u})\rangle_{i}=f_{i, l o w}<(\mathbf{p}-\mathbf{S u})_{i}<f_{i, u p} \\
& \langle(\mathbf{p}-\mathbf{S u})\rangle_{j}=\left|(\mathbf{p}-\mathbf{S u})_{j}\right|<\mu(\mathbf{p}-\mathbf{S u})_{i}
\end{aligned}
$$

In Equations (4)-(6), $\mathbf{u}$ is the total structure displacement vector $\left(\mathbf{u}^{T}=\left[\begin{array}{lll}\mathbf{u}_{i} & \mathbf{u}_{j} & \boldsymbol{\Phi}_{k}\end{array}\right]\right.$, in which $\mathbf{u}_{i}$ and $\mathbf{u}_{j}$ consist of the translational displacements along $z$ and $x$, respectively, whereas $\boldsymbol{\Phi}_{k}$ contains rotations of the finite element nodes), $\mathbf{u}^{\text {cat }}$ is the greenfield surface displacement due to tunnelling, $\mathbf{p}$ is the external loading vector of the structure, $\mathbf{S}$ is the structure 
(a)

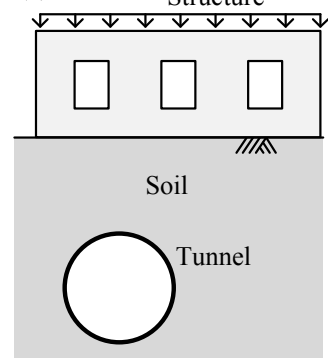

(b)

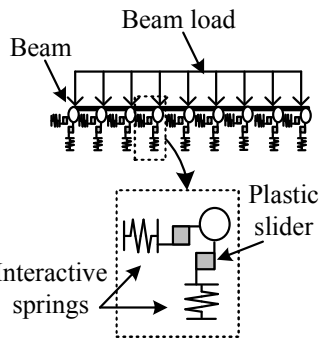

Fig. 2. (a) sketch of the problem; (b) mechanical representation of the model.

stiffness matrix, $\boldsymbol{\Lambda}$ is the soil flexibility matrix defined with the elastic integrated forms of Mindlin's solutions given by Vaziri et al. (1982), $\boldsymbol{\Lambda}^{l}$ is the local soil flexibility given by the diagonal matrix of $\boldsymbol{\Lambda}, \boldsymbol{\Lambda}^{*}$ is soil flexibility matrix without the main diagonal, $\mathbf{K}^{*}=\left(\boldsymbol{\Lambda}^{l}\right)^{-1}$ is the local stiffness matrix of the soil, $f_{i, u p}$ and $f_{i, l o w}$ are the upper and lower limit loads of the vertical plastic sliders, and $\mu$ is the friction coefficient between the soil and foundation. In this study, $f_{i, l o w}=0$ and $f_{i, u p}=\infty$ (i.e. for vertical springs, linear elastic behaviour in compression and no tensile strength). The reaction forces applied by the soil to the foundation nodes are $\mathbf{F}=\mathbf{S u}-\mathbf{p}$, whereas the compatibility condition requires that the displacement vector $\mathbf{u}=\mathbf{u}^{c}+\mathbf{u}^{s l}$, in which $\mathbf{u}^{c}$ is the soil continuum displacements and $\mathbf{u}^{s l}$ is the slider displacements. An iterative and incremental procedure is needed to solve Equations (4)-(6) (Franza \& DeJong, 2018).

A fully elastic method based on the perfect soil-structure bonding condition is obtained by imposing $\mathbf{u}^{s l}=0$. In this case, Equation (4) can be written as Equation (7). Because of the superposition principle, tunnelling-induced displacements can be calculated assuming $\mathbf{p}=0$.

$$
\left(\mathbf{S}+\mathbf{K}^{*}+\mathbf{K}^{*} \boldsymbol{\Lambda}^{*} \mathbf{S}\right) \mathbf{u}=\mathbf{p}+\mathbf{K}^{*} \boldsymbol{\Lambda}^{*} \mathbf{p}+\mathbf{K}^{*} \mathbf{u}^{c a t}
$$

In this study, purely elastic and elastoplastic solutions are referred to as 'EL' and 'EP', respectively.

\section{Tunnelling-induced structure deformations and the role of soil-structure interaction}

Tunnelling-induced structural damage is often assessed by assuming that the structure deforms according to the greenfield conditions. A maximum building tensile strain, $\varepsilon_{\max }$, is then calculated with deep beam theory; $\varepsilon_{\max }$ depends on bending strain $\left(\varepsilon_{b}\right)$, diagonal strain $\left(\varepsilon_{d}\right)$, and average horizontal axial strain $\left(\varepsilon_{h}\right)$ of the structure (Burland \& Wroth, 1974; Boscardin \& Cording, 1989; Mair et al., 1996).

$$
\varepsilon_{b}=\frac{D R}{\left(\frac{B_{r}}{12 t}+\frac{3 I}{2 t B_{r} H} \frac{E}{G}\right)} \quad \varepsilon_{d}=\frac{D R}{\left(1+\frac{H B_{r}^{2}}{18 I} \frac{G}{E}\right)}
$$

where $H$ is the height of the building, $B_{r}$ is the building transverse length in the sagging or hogging region (indicated as $B_{\text {sag,bld }}$ and $B_{h o g, b l d}$, respectively, in Figure 3), $E$ and $G$ are the Young's and shear moduli, $I$ is the second moment of area of the idealised beam, $t$ is the maximum distance between the neutral axis and the edge of the beam in tension, and $D R$ is the deflection ratio (defined in Figure 3). The structure transverse length and location with respect to the tunnel centreline can be defined in terms of $D_{u}$ and $D_{l}$, which are discussed later in the text.

Stiff structures react to ground displacements and deform less than a fully-flexible structures. To quantify this soilstructure interaction (SSI), modification factors for the deflection ratio, $M^{D R, s a g}$ an $M^{D R, h o g}$, were proposed by Potts \& Addenbrooke (1997) as follows

$$
M^{D R, \text { sag } / \text { hog }}=\frac{D R_{\text {sag } / \text { hog }, \text { bld }}}{D R_{\text {sag } / \text { hog }, g f}}
$$

where building deflection ratios are $D R_{\text {sag,bld }}$ and $D R_{h o g, b l d}$ (depending on the structure inflection point, $i_{b l d}$ ), deflection ratios of the greenfield settlement trough are $D R_{s a g, g f}$ and $D R_{h o g, g f}$ (determined by the greenfield inflection point position, $i)$.

Structures with continuous horizontal foundation elements have a significant axial stiffness and, consequently, tunnellinginduced structural horizontal strains $\varepsilon_{h}$ are generally low (Burland et al., 2004; Dimmock \& Mair, 2008). Similar to the $D R$ s, Potts \& Addenbrooke (1997) proposed that the modification factors for the average horizontal strain can be defined 


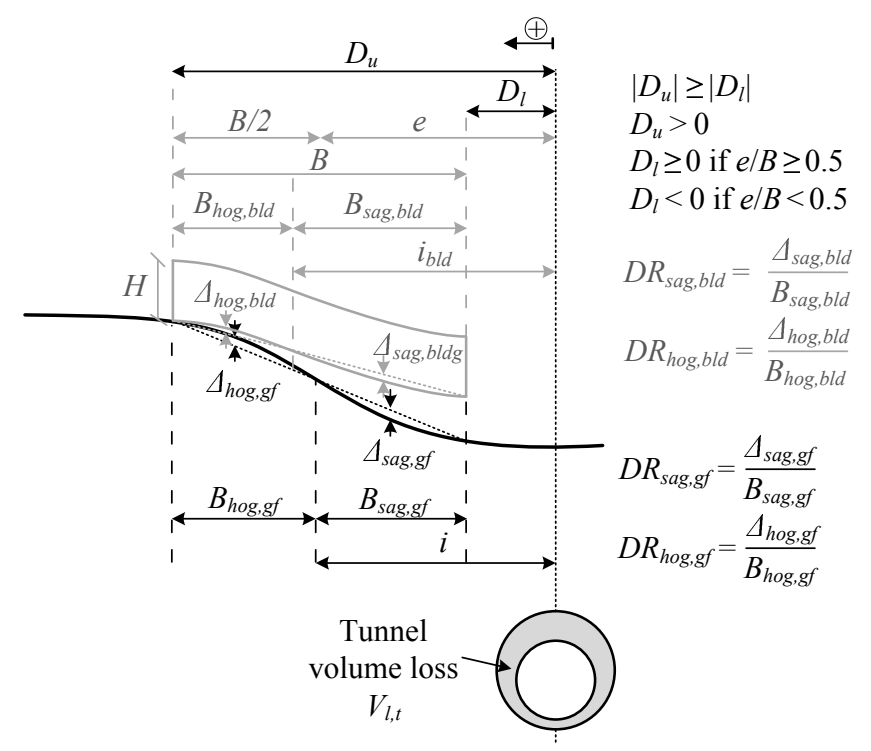

Fig. 3. Nomenclature for the flexural TSI problem.

as follows

$$
M^{\varepsilon h, s a g / h o g}=\frac{\varepsilon_{h, s a g / h o g, b l d}}{\varepsilon_{h, s a g / h o g, g f}}
$$

where $\varepsilon_{h, s a g / h o g, b l d}$ and $\varepsilon_{h, s a g / h o g, g f}$ are computed, respectively, within $B_{\text {sag/hog,bld }}$ and $B_{\text {sag/hog,gf }}$.

Methods based on the relative structure-soil stiffness have been proposed to assess $M^{D R}$. For example, Franzius et al. (2006) and Mair (2013) defined, respectively, $\rho_{m o d}^{*}$ and $\rho_{\text {sag }}, \rho_{\text {hog }}$ as dimensionless relative stiffness factors that are expressed as

$$
\rho_{\text {mod }}^{*}=\frac{E I}{E_{s} z_{t} B^{2} L}=\frac{E I^{*}}{E_{s} z_{t} B^{2}}
$$

西

$$
\rho_{\text {sag } / \text { hog }}=\frac{E I}{E_{s} B_{\text {sag } / \text { hog }, g f}^{3} L}=\frac{E I^{*}}{E_{s} B_{\text {sag } / \text { hog }, g f}^{3}}
$$

where $E I$ is the bending stiffness of the superstructure (in $\mathrm{kN} \mathrm{m}^{2}$ ), $E I^{*}$ is $E I$ per running metre (in $\mathrm{kN} \mathrm{m}^{2} / \mathrm{m}$ ), $E_{s}$ is a soil Young's modulus representative of the soil affected by the excavation (accounting for the average elastic modulus of the soil above the tunnel and the soil stiffness degradation with strain level), $L$ is the longitudinal length of the building in the tunnel axis direction, $B_{\text {sag,gf }}$ and $B_{\text {hog,gf }}$ are the transverse lengths of the building in the sagging and hogging zones based on the greenfield settlement trough.

Franzius et al. (2006) and Mair (2013) proposed design charts in which $M^{D R}$ are related to $\rho_{\text {mod }}^{*}$ and $\rho_{\text {sag }}, \rho_{\text {hog }}$, respectively. Franzius et al. (2006) envelopes vary with the normalised tunnel-structure eccentricity $e / B$ (see Figure 3 ) and deformation zone. However, $\rho_{\text {mod }}^{*}$ does not account for the shape of the greenfield settlement trough that is related to $i$, although Franzius et al. (2006) results displayed that, for a given structure, $M^{D R}$ increases with tunnel depth $z_{t}$ (thus $i$ ).

Mair (2013) proposed $\rho_{\text {sag }}$ and $\rho_{\text {hog }}$ and the same design relationship $M^{D R}-\rho$ in sagging and hogging zones. In addition, $B_{\text {sag }, g f}$ and $B_{\text {hog,gf }}$ relate to $i$ and thus $\rho_{\text {sag }}$ and $\rho_{\text {hog }}$ indirectly account for the shape of the settlement trough (consequently, $\rho_{\text {sag }}$ and $\rho_{\text {hog }}$ could be used for both tunnelling in clays and sands). The definition of $\rho_{\text {sag }}$ and $\rho_{\text {hog }}$ implies that a structure spanning both hogging and sagging zones responds to tunnelling as independent structures in each deformation zone. However, structural stiffness can modify the shape of the building settlement curve; consequently, the locations of the inflection points of the structure $\left(i_{b l d}\right)$ and the greenfield settlement trough $(i)$ may differ, as shown in Figure 3 (Farrell et al., 2014; Frischmann et al., 1994; Lu et al., 2001; Potts \& Addenbrooke, 1997; Taylor \& Yip, 2001).

Equation (12) was modified by Giardina et al. (2015) to account for the structure weight effects on $M^{D R}$; however, Bilotta et al. (2017), Franzius et al. (2004), Giardina et al. (2015), and Franza \& DeJong (2018) concluded that the influence of the self-weight on $M^{D R}$, while important in some cases, is generally secondary compared to the role of bending stiffness for ordinary structures and low-medium volume losses.

Finally, as displayed by Equation (8), tensile strains depend on the building transverse length $B_{r}=B_{\text {sag,bld }}$ or $B_{\text {hog,bld }}$. Therefore, assuming $B_{\text {sag } / \text { hog }, \text { bld }}=B_{\text {sag } / \text { hog,gf }}$ (implying $i_{\text {bld }}=i$ ) can lead to erroneous damage assessment. To account for the variation in shape of the building settlement curve, the modification factors $M^{B, s a g}$ and $M^{B, h o g}$ for the sagging and 
hogging region length are defined as follows

$$
M^{B, s a g / h o g}=\frac{B_{\text {sag } / \text { hog }, \text { bld }}}{B_{\text {sag } / h o g, g f}}
$$

$M^{B}$ has received less attention in the literature than $M^{D R}$. Farrell (2010) suggested an empirical correlation between $i_{b l d} / i$ and structure-soil stiffness, whereas Potts \& Addenbrooke (1997) and Franza et al. (2017) suggested that structure deflection shape is dependent on shear deformability and structural configuration, respectively, which are related.

\section{COMPARISON OF CONTINUUM SOLUTION PREDICTIONS WITH CENTRIFUGE DATA}

Results from the centrifuge test series performed by Ritter et al. (2017a,b) at $75 \mathrm{~g}$ were considered. In the following, both model dimensions and results are reported in prototype scale. $1 / 75$ th scale models, each consisting of a bearing wall structure with openings on strip footings affected by tunnelling, were tested (see Figure 4). The tunnel was excavated in uniform dense dry silica sand (soil relative density $I_{d}=90 \%$ ) and simulated with a plane-strain model tunnel. At prototype scale, a $6.2 \mathrm{~m}$ diameter $(D)$ tunnel with a cover-to-diameter ratio $C / D=1.3$ and a depth to tunnel axis $z_{t}=11.3 \mathrm{~m}$ was modelled. The model structure was obtained through $3 \mathrm{D}$ printing of a material with properties similar to masonry. The structure was orthogonal to the tunnel. The opening ratio, $O$, of the transverse walls (simulating window distributions) ranged between $20 \%$ and $40 \%$. Only external walls were supported by strip footings (see Figure 4(b)); this structural detail is taken into account in this paper considering the ratio $\chi$ between the soil-foundation contact surface $A_{f}$ (i.e. the area of the footing) and the area of the building $A_{s}=B \times L$. Model structures were located both centrally and eccentrically with respect to the tunnel centreline (corresponding to $x=0$ ) with a normalised eccentricity $e / B$ varying between 0 and 0.8 . The average stress at the contact area between strip footings, $A_{f}$, and soil was $\sigma=80 \mathrm{kPa}$.

$E I$ and $E A$ of the entire structure were estimated as the summation of the contributions of the walls and foundation elements in the transverse direction to the tunnel axis (see dashed lines in Figure 4(c)); computed $E I$ and $E A$ values are reported in Table 1, normalised by the longitudinal structure length $L$. The second moment of area $I$ of each element was taken relative to the structure neutral axis for pure bending deformations (e.g. the geometric centroid of the full crosssection composed of wall and foundation for a uniform material). In the following, the term 'neutral axis' is used to indicate the axis where the longitudinal strain is zero for pure bending deformations; however, there can be longitudinal strains at the 'neutral axis' due to axial deformations. To account for the openings, $E I$ and $E A$ of the bearing walls were computed by decreasing the cross-section values, respectively, by the reduction factor $\alpha$ given by Melis \& Rodriguez Ortiz (2001) and $\lambda$, which is the ratio between average cross-sectional area over the building transverse length $B$ and full cross-section area. For $O=20$ and $40 \%, \alpha=0.60,0.15$ whereas $\lambda=0.80,0.60$, respectively. The stiffening effect of transverse walls was neglected because the main structural deflection is obtained in the transverse plane. It should be also noted that $\alpha$ and $\lambda$ do not account for the effects of the opening shape and their distribution.

Previous research mostly investigated the TSI by adopting equivalent beam and plate models of the structure (Potts \& Addenbrooke, 1997; Franzius et al., 2006; Farrell et al., 2014; Namazi \& Mohamad, 2013a). These equivalent models are fully representative for buildings with a raft foundation because the entire equivalent structure is in contact and interacts with the underlying soil; consequently, $E I^{*}=E I / L$ and $E A^{*}=E A / L$ are well defined for raft foundations. However, average stiffness values $(E I / L, E A / L)$ reported in Table 1 neglect that in the centrifuge tests the foundation consists of strip footings and that, consequently, only the soil directly beneath the foundation elements is interacting with the structure. To incorporate this foundation scheme, bending and axial stiffness per metre run $\left(E I^{*}, E A^{*}\right)$ are estimated as follows

$$
E I^{*}=\frac{E I}{\chi L}\left[\mathrm{kNm}^{2} / \mathrm{m}\right] \quad E A^{*}=\frac{E A}{\chi L}[\mathrm{kN} / \mathrm{m}]
$$

where $\chi=1$ for raft foundations and $\chi=A_{f} / A_{s} \leq 1$ for strip and separated footings (see Figure 4(b)). However, this approximation has limitations because it does not account for the shape of the 3D foundation scheme and the location of the footings with respect to the tunnel axis.

Finally, it should be noted that for the considered buildings on continuous strip footings, $E I^{*}=E I_{\text {facade }} / d_{\text {foot }}$ could have been an alternative approach, where $E I_{\text {facade }}$ is the facade stiffness and $d_{\text {foot }}$ is the footing cross-sectional width in the longitudinal direction. However, for the centrifuge buildings, this approach neglects the presence of the longitudinal footings and would overestimate the structure stiffness with respect to the soil.

For the elastic continuum, a Young's modulus $E_{s}=45 \mathrm{MPa}$ and a Poisson's ratio $\nu_{s}=0.25$ were assumed; this $E_{s}$ value represents a lower bound estimation for the simulated soil conditions at $V_{l, t} \leq 2 \%$ (Ritter, 2017). The friction coefficient $\mu$ 


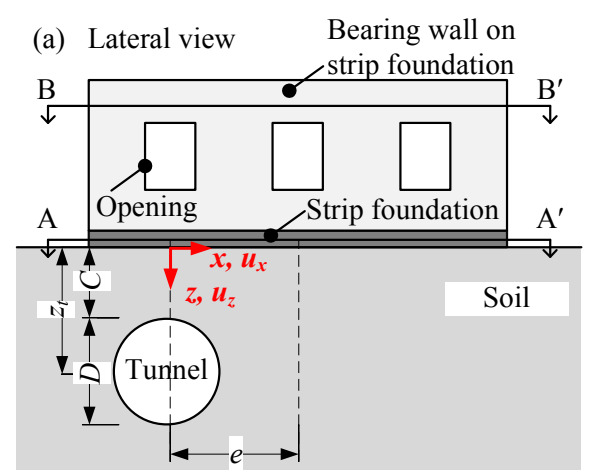

(b) A-A' Plan view

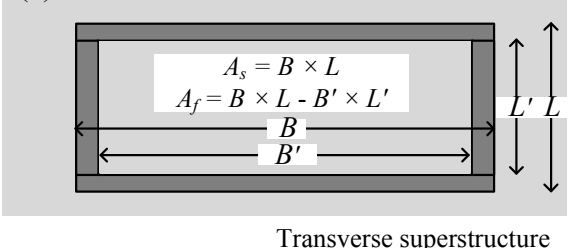

(c) B-B' Plan view and foundation elements

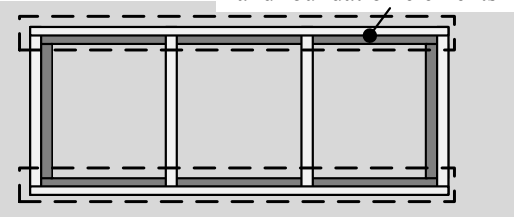

Fig. 4. Configuration modelled in centrifuge tests.

Table 1. Centrifuge test series of TSI (in prototype scale).

\begin{tabular}{ccccccc}
\hline $\begin{array}{c}\text { Label } \\
\text { Test }\end{array}$ & $\begin{array}{c}B \\
(\mathrm{~m})\end{array}$ & $\begin{array}{c}e / B \\
(-)\end{array}$ & $\begin{array}{c}O \\
(\%)\end{array}$ & $\begin{array}{c}E A / L \dagger \\
(\mathrm{kN} / \mathrm{m})\end{array}$ & $\begin{array}{c}E I / L \dagger \\
(\mathrm{kNm})\end{array}$ & $\begin{array}{c}\chi \\
(-)\end{array}$ \\
\hline SR-A & 15.0 & 0 & 20 & $4.510^{5}$ & $1.510^{6}$ & 0.30 \\
SR-B & 15.0 & 0.8 & 20 & $4.010^{5}$ & $1.310^{6}$ & 0.30 \\
SR-C & 15.0 & 0.5 & 20 & $3.710^{5}$ & $1.210^{6}$ & 0.30 \\
SR-D & 15.0 & 0.8 & 40 & $2.010^{5}$ & $4.010^{5}$ & 0.30 \\
SR-E & 19.5 & 0.5 & 20 & $3.510^{5}$ & $1.110^{6}$ & 0.28 \\
SR-F & 19.5 & 0.5 & 40 & $4.110^{5}$ & $8.110^{5}$ & 0.28 \\
\hline$L=7.5 m ; \sigma=80 \mathrm{kPa}$ \\
\hline
\end{tabular}

between the foundation and the continuum was specified to be $\tan \left(30^{\circ}\right)$. The input displacement vector $\mathbf{u}^{\text {cat }}$ was defined on the basis of the greenfield centrifuge test from Marshall et al. (2012) by curve-fitting surface measurements of soil $u_{z}$ and $u_{x}$ with Equations (2) and (3).

The characteristics of the implemented equivalent simple beams are reported in Table 2. The subscript $m$ was added to indicate the properties of the equivalent beam model. The equivalent beams had a finite element size of $0.5 \mathrm{~m}$ and a transverse length matching the corresponding prototype structure $B$. The Young's moduli, $E_{m}$, and cross-sectional dimensions (depth, $d_{b, m}$, and width, $\left.b_{b, m}=1 \mathrm{~m}\right)$ of the equivalent simple beams were set to match the cross-sectional properties $E_{m} I_{m}^{*}$ and $E_{m} A_{m}^{*}$ with the prototype $E I^{*}$ and $E A^{*}$ of the centrifuge model buildings $\left(E_{m} A_{m}^{*}=E A^{*}, E_{m} I_{m}^{*}=E I^{*}\right)$. Two equivalent simple beams with varying cross-sectional properties were adopted for a given centrifuge test to investigate the influence of the ratio $\chi$ of the target building in TSI analyses. As shown in Table 2, two simulation series, labelled 'SRs' and 'SRf', were carried out with $\left(E_{m} I_{m}^{*} / 1, E_{m} A_{m}^{*} / 1\right)$ equal to $(E I / L, E A / L)$ and $(E I /(\chi L), E A /(\chi L))$, respectively. Finally, a uniform vertical load $q_{z, m}=\sigma \times b_{b, m}=80 \mathrm{kN} / \mathrm{m}$ was adopted.

Firstly, tunnelling-induced vertical displacements $\left(u_{z}\right)$ of equivalent beams SRf, predicted by the elastic EL (black dashed lines) and elastoplastic EP (black solid lines) solutions, are compared with the centrifuge measurements in Figures 5 and 6 for $V_{l, t}=1,2 \%$. Note that centrifuge displacements are reported both at the foundation level $\left(z=0\right.$ indicated as $\left.z_{g}\right)$ and at the height of the neutral axis of the structure $\left(z=-2.5 \mathrm{~m}\right.$ indicated as $\left.z_{b}\right)$, which was computed considering the strip foundation and the bearing wall as a composite beam. Furthermore, plastic slider displacements from EP solutions (black markers) as well as contact stresses $\sigma$ between the equivalent beam and the soil (grey lines) are plotted. Greenfield settlements are 
Table 2. Equivalent simple beams.

\begin{tabular}{lccccc}
\hline Label & $\begin{array}{c}B_{m} \\
(\mathrm{~m})\end{array}$ & $\begin{array}{c}d_{b, m} \\
(\mathrm{~m})\end{array}$ & $\begin{array}{c}E_{m} \\
(\mathrm{~Pa})\end{array}$ & Target & $\begin{array}{c}E_{m} I_{m} / 1, \\
E_{m} A_{m} / 1\end{array}$ \\
\hline SRs-A & 15 & 6.3 & $7.110^{7}$ & SR-A & $E I / L$, \\
SRs-B & 15 & 6.2 & $6.510^{7}$ & SR-B & $E A / L$ \\
SRs-C & 15 & 6.3 & $5.810^{7}$ & SR-C & \\
SRs-D & 15 & 4.9 & $4.210^{7}$ & SR-D & \\
SRs-E & 19.5 & 6.2 & $5.610^{7}$ & SR-E & \\
SRs-F & 19.5 & 4.9 & $8.310^{7}$ & SR-F & \\
\hline SRf-A & 15 & 6.3 & $2.410^{8}$ & SR-A & $E I /(\chi L)$, \\
SRf-B & 15 & 6.2 & $2.210^{8}$ & SR-B & $E A /(\chi L)$ \\
SRf-C & 15 & 6.3 & $2.010^{8}$ & SR-C & \\
SRf-D & 15 & 4.9 & $1.410^{8}$ & SR-D & \\
SRf-E & 19.5 & 6.2 & $2.010^{8}$ & SR-E & \\
SRf-F & 19.5 & 4.9 & $3.010^{8}$ & SR-F & \\
\hline \multicolumn{7}{l}{$b_{b, m=1 \mathrm{~m} ;} q_{z}=80 \mathrm{kN} / \mathrm{m}$} \\
\hline \multicolumn{7}{c}{}
\end{tabular}

also displayed (dotted lines). Finally, acronyms are used in the legend: STR for the structure; SLD for the slider; EXP for experimental results.

Regarding centrifuge test outcomes, settlement data at the two heights $z_{g}$ and $z_{b}$ are nearly identical (the difference is within the error in the experimental measurement). In addition, the eccentric structures with $e / B>0$ in Figures 5 (b)-(f) and 6(b)-(f) displayed a semi-flexible response and greater settlements than greenfield curves, particularly at high volume losses; this is in agreement with numerical analyses performed in previous works (Bilotta et al., 2017; Burd et al., 2000; Franzius et al., 2004). Meanwhile, the central structure SR-A with $e / B=0$ in Figure 6(a) underwent small deflections with respect to greenfield curves.

Although EL and EP solutions cannot capture the increased level of settlements due to the structure weight, they generally match the structure deflections from the centrifuge tests reasonably well. Additionally, the EL and EP solutions are identical for all structures except for SRf-A in Figure 6(a), for which EP predicted $u^{s l}$ greater than zero (this agrees with centrifuge tests: gap formation occurred above $V_{l, t} \approx 1.5 \%$ ); in the other scenarios, the beam stiffness was not sufficiently stiff to result in the complete unloading of the soil. This statement is supported by the plot of the contact stresses $\sigma$, which reach null values over a significant transverse length only in Figures 5(a) and 6(a).

Finally, the variation in the soil-beam contact stress profile $(\sigma)$, which is due to tunnelling-induced load redistribution along the structure, is considered in order to provide further insights into TSI. The stress results in Figures 6(a), (b) and (c) (structures with the same transverse length $B$ and opening percentage $O$ ) show that [i] load redistribution varied with $e / B$, which indicates a different SSI mechanism, and [ii] increased $e / B$ reduced the magnitude of the load redistribution.

In Figures 7 and 8, horizontal greenfield and structure displacement profiles $\left(u_{x}\right)$ are displayed with a layout consistent to Figures 5 and 6; slider displacements are indicative of beam-soil slippage rather than gap formation. For SR-F visible cracking occurred at $V_{l, t}=2.6 \%$ (Ritter, 2017) and potential micro-cracking might have occurred at even lower volume loss values (see Figures $6(\mathrm{f})$ and $8(\mathrm{f})$ ), whereas cracking started at a greater $V_{l, t}$ in the other tests. Consequently, material nonlinearity should be relatively insignificant for the volume loss range of interest.

Although tunnel engineers often measure structure axial deformations with monitoring systems placed near the ground level, experimental data show qualitative differences between structure displacements $u_{x}$ at the ground level, $z_{g}$, and the neutral axis height, $z_{b}$ (compare light and dark markers). In particular, these displacement profiles are associated with different horizontal strains $\varepsilon_{h}=u_{x}^{\prime}(x, z)$ (i.e. $u_{x}^{\prime}\left(x, z_{g}\right) \neq u_{x}^{\prime}\left(x, z_{b}\right)$ ). Considering that [i] shear deformations do not result in $\varepsilon_{h}$ and [ii] tilting effects on $\varepsilon_{h}$ (due to the projection of the vertical self-weight along the tilted structure axis) are negligible for the modelled buildings (Namazi \& Mohamad, 2013b), the variation in $\varepsilon_{h}$ at the structure bottom $z_{g}$ with respect to the level $z_{b}$ was due the bending deformations. For instance, centrifuge data in Figure 8(e) show shortening of the structure fibres at $z_{g}$ with respect to $z_{b}$ level data. Consequently, when possible (e.g. during experimental testing), horizontal strain $\varepsilon_{h}$ should be measured at the height of the structure's neutral axis.

Centrifuge data of structures with $e / B>0$ shown in Figures 7(b)-(f) and 8(b)-(f) indicate that the structure is significantly dragged towards the tunnel centreline while horizontal strains tend to remain small and tensile (except in sub-plot(c)). On the other hand, the central structure SR-A with $e / B=0$ was compressed by horizontal soil movements caused by tunnelling, and experienced negligible average $u_{x}$ because of symmetry.

Taking into consideration the influence of bending on $u_{x}$, the EP predictions are compared in terms of overall shift (i.e. average displacements $u_{x}$ over the building transverse length $B$ ) with the centrifuge $u_{x}$ at the ground level $z_{g}$, whereas the horizontal strain level of the equivalent beam are compared with experimental $\varepsilon_{h}$ at $z_{b}$. SRf beams (accounting for 

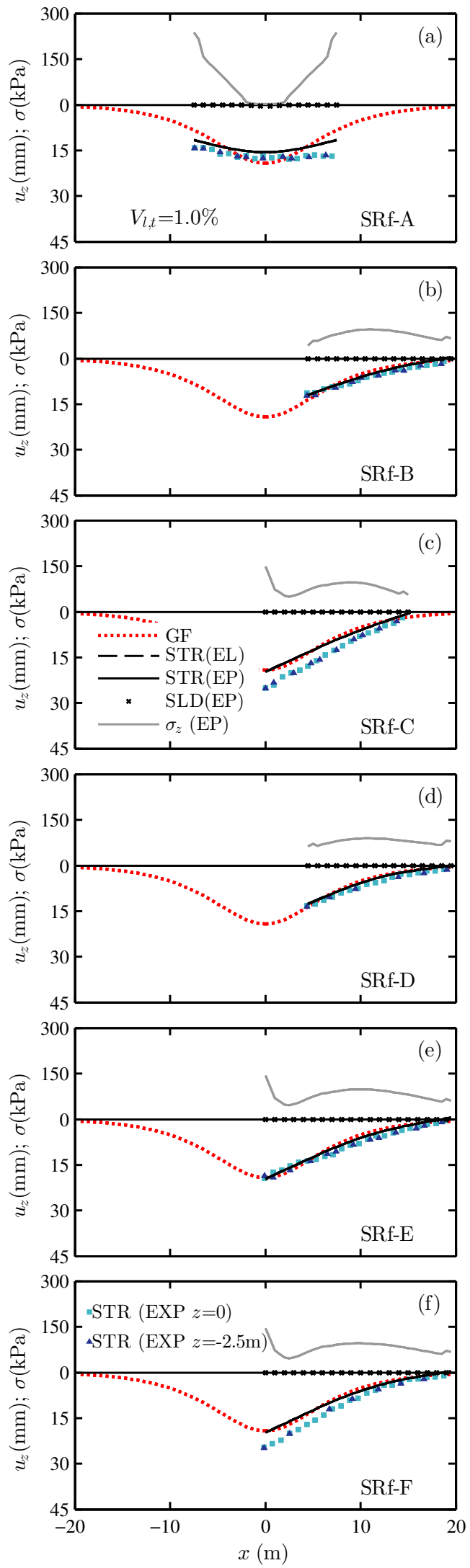

Fig. 5. Settlements of the building and in greenfield conditions as well as gap formation and contact stresses between the beam and the soil for $V_{l, t}=1 \%$.

the ratio $\chi$ ) provide a good prediction of horizontal displacements and strains compared with centrifuge tests, except for SR-C in Figures 7(c) and 8(c). During test SR-C, the bearing wall underwent significant axial compression above the tunnel 

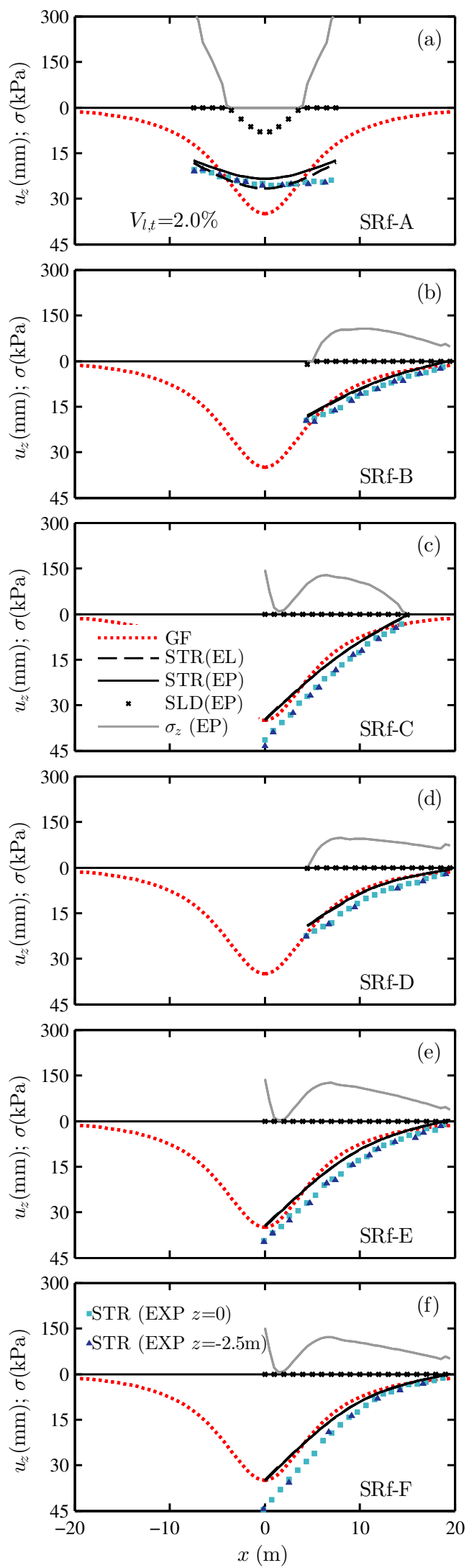

Fig. 6. Settlements of the building and in greenfield conditions as well as gap formation and contact stresses between the beam and the soil for $V_{l, t}=2 \%$. 

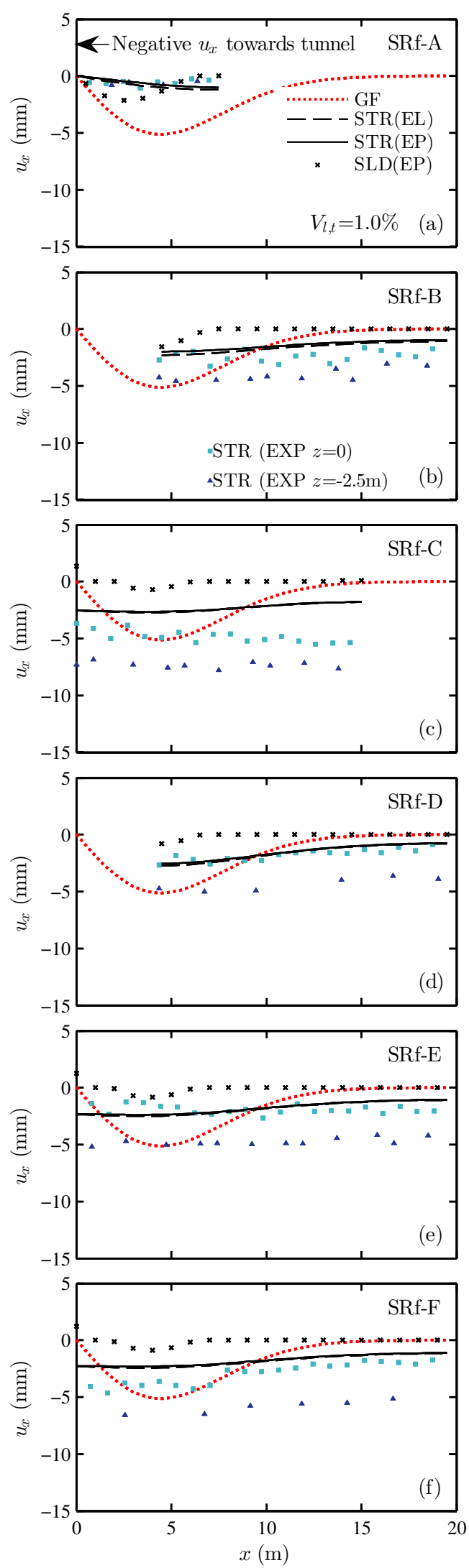

Fig. 7. Horizontal displacements of the building and in greenfield conditions as well as slippage between the beam and the soil for $V_{l, t}=2 \%$.

Finally, Figure 8 indicates that allowing for slippage in the EP solution resulted in lower axial strains with respect to the EL method, though the difference between the EP and EL results is relatively small. 

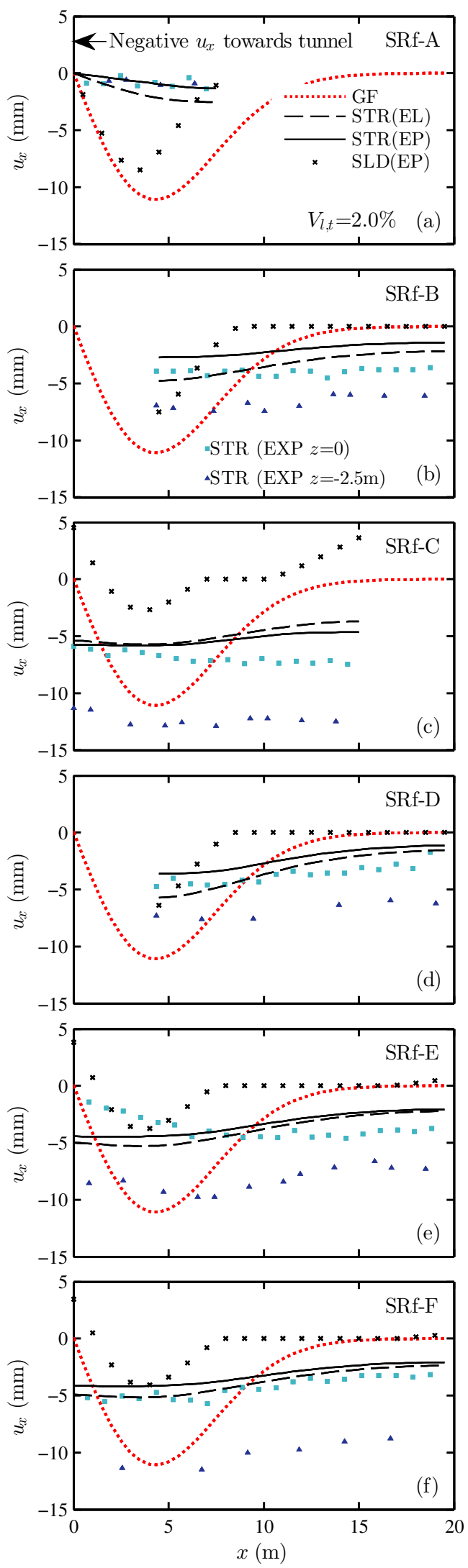

Fig. 8. Horizontal displacements of the building and in greenfield conditions as well as slippage between the beam and the soil for $V_{l, t}=2 \%$.

Next, modification factors for the deflection ratio $\left(M^{D R}\right)$ and average horizontal strain $\left(M^{\varepsilon h}\right)$ in the sagging and hogging regions for both centrifuge tests and EP solutions are shown in Figure 9. The plots provide information on the effects of $\chi$ by comparing continuum solutions for SRs and SRf structure (dashed and solid lines, respectively). 

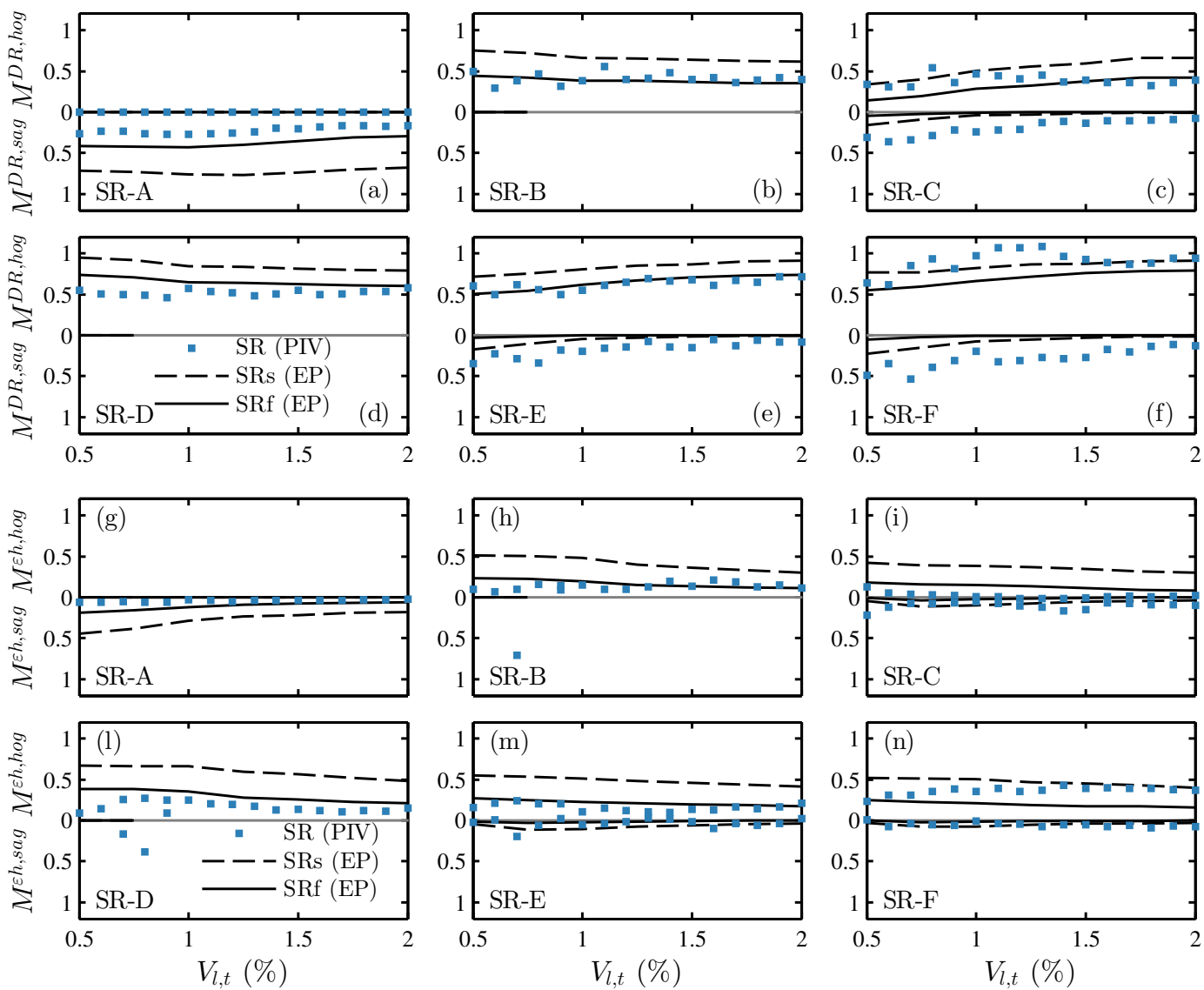

Fig. 9. Comparison between experimental and computed modification factor against $V_{l, t}:$ (a)-(f) $M^{D R},(\mathrm{~g})-(\mathrm{h}) M^{\varepsilon h}$.

Firstly, $M^{D R}$ in Figures 9(a)-(f) are analysed. Solutions implementing SRf equivalent simple beams predicted $M^{D R, s a g}$ for the central structure $(e / B=0)$ and $M^{D R, h o g}$ for eccentric structures $(e / B \geq 0.5)$ that are in good agreement with centrifuge data, except for the underestimation of hogging deformations of the longest structure SR-F in Figures $9(\mathrm{f})$. These errors were potentially due to the effects of the structure shear deformability. In addition, although SRf models slightly underestimated $M^{D R, s a g}$ for $e / B \geq 0.5$ (Figures $9(\mathrm{~b})$-(f)), SRf beams captured that the response to tunnelling for eccentric building is more flexible in the hogging region than in the sagging zone (i.e. for $e / B \geq 0.5, M^{D R, h o g}>M^{D R, s a g}$ ). On the other hand, results in Figures 9(a)-(e) indicate that the use of simple beams SRs associated with $\chi=1$ (dashed lines) resulted in the overestimation of $M^{D R, s a g}$ and $M^{D R, h o g}$ for central and eccentric buildings, respectively. Also note that adopting $E_{s}$ higher than $45 \mathrm{MPa}$ (the adopted lower bound value) would result in $M^{D R}$ values greater than shown in Figure 9. Thus, the results indicate that accounting for the ratio $\chi$ provides a more accurate prediction.

In Figures 9(g)-(n), EP solutions with SRf equivalent beams provided $M^{\varepsilon h}$ values in agreement with centrifuge results, except for the underestimation of $M^{\varepsilon h, h o g}$ for SRf-F in Figure $9(\mathrm{n})$. Despite this, it should be noted that centrifuge structure SR-F had an average $\varepsilon_{h, h o g}$ limited in magnitude (for instance, building $\varepsilon_{h, h o g}<+0.03 \%$ at $V_{l, t}=2 \%$ ); thus, the error resulting from the underestimation of $M^{\varepsilon h, h o g}$ in Figure $9(\mathrm{n})$ in terms of structure tensile strains would be small. On the other hand, $M^{\varepsilon h}$ associated with SRs structures were greater than benchmark centrifuge data; thus, assuming $E A^{*}$ from $\chi=1$ underestimated relative structure-soil stiffness resulting in significant horizontal strains within the equivalent beam.

\section{EFFECTS OF STRUCTURE CONTINUITY ACROSS SAGGING AND HOGGING GREENFIELD REGIONS}

To investigate the effects of structure continuity (i.e. the interaction between the sagging and hogging portions of the structure) on the TSI mechanisms, structures with the same cross-sectional stiffness, but with different transverse length and eccentricity, were simulated. Greenfield ground movements and geotechnical parameters were assumed as in the previous section. A summary of the analysed scenarios is reported in Table 3. Structures ST-SS and ST-H were predominantly located in the sagging and hogging regions of the greenfield settlement trough, respectively. Structures ST-SSH and ST-HSSH were obtained by extending beams ST-SS in the hogging zone on one or both sides respectively, whereas the structures ST-SH 
and ST-SSH are extended towards the sagging zone with respect to ST-H. The letters 'S' and 'H' indicate the sequence of sagging and hogging regions across the transverse length of the beam.

Table 3. Simple beams used to investigate structure continuity effects.

\begin{tabular}{lcccc}
\hline Label & $\begin{array}{c}B_{m} \\
(\mathrm{~m})\end{array}$ & $\begin{array}{c}e / B \\
(-)\end{array}$ & $\begin{array}{c}d_{b, m} \\
(\mathrm{~m})\end{array}$ & $\begin{array}{c}E_{m} \\
(\mathrm{~Pa})\end{array}$ \\
\hline ST-SS & 15 & 0 & 6.0 & $2.010^{8}$ \\
ST-SSH & 27.5 & 0.23 & 6.0 & $2.010^{8}$ \\
ST-HSSH & 40 & 0 & 6.0 & $2.010^{8}$ \\
ST-H & 15 & 0.8 & 6.0 & $2.010^{8}$ \\
ST-SH & 19.5 & 0.5 & 6.0 & $2.010^{8}$ \\
\hline$b_{b, m}=1 \mathrm{~m} ; q_{z}=80 \mathrm{kN} / \mathrm{m}$ & & & \\
\hline
\end{tabular}

Settlements, $u_{z}$, of the simple beams in Table 3 predicted by EL and EP solutions are displayed in Figure $10 . M^{D R}$ and $B_{r}$ of the sagging and hogging deformation zones associated with greenfield curves and EL analyses are shown in Figures 11 and 12. Results in Figures 10(a), (b), (c) and 10(b), (d), (e) illustrate, respectively, the effects of the extension of structure towards the greenfield hogging and sagging region. In the following, the terms 'primary' and 'secondary' deformation modes are used as follows: for $e / B=0-0.3$, primary $=$ sagging and secondary $=$ hogging; for $e / B \geq 0.5$, primary $=$ hogging and secondary $=$ sagging.

Firstly, the interaction mechanisms associated with structures ST-SS/SSH/HSSH are analysed, for which sagging is the primary deformation mode. Settlements in Figures 10(a), (b) and (c) display that, for structures with $e / B=0-0.3$, the additional transverse length $B_{\text {hog,gf }}$ of the structure in the greenfield hogging region slightly decreased structure settlements because of the constraint given by the soil undergoing low tunnelling-induced settlements (at $x>10 \mathrm{~m}$ ). This is confirmed by the observed stress redistribution. Then, comparing greenfield settlement troughs and building displacements for ST-SSH and ST-HSSH in Figures 10(b) and (c), structural stiffness resulted in $i_{b l d}>i$; consequently, the structure transverse length of sagging deflection, $B_{\text {sag,bld }}$, was greater than the greenfield value, $B_{\text {sag,gf }}$ (as confirmed by data in Figure 11(b)). In addition, the $M^{D R, s a g}$ values shown in Figure 11(a) were significantly affected by the extension of the structure into the greenfield hogging region. Larger values of $B_{\text {sag,bld }}$ in Figure 11(b) correspond to an increase in $M^{D R, s a g}$. For structures primarily in sagging, the increase in the structure transverse length in the greenfield hogging zone $\left(B_{h o g, g f}\right)$ decreased the relative structure-soil stiffness for sagging distortions and induced larger $M^{D R, s a g}$. This interaction mechanism is not accounted for in Equation (12). Furthermore, the fact that $B_{\text {hog, bld }}<B_{\text {hog }}$ in Figure 11(b) for ST-SSH/HSSH indicates that the stiffness of the structure was sufficient to decrease the building hogging transverse length.

Next, the interaction mechanisms of structures ST-H/SH/SSH are addressed. Figure 10(e) displays that ST-SH $(e / B=0.5$, hogging is the primary deformation mode) undergoes mostly hogging deformations despite a mixed sagginghogging greenfield settlement trough (in Figure $12(\mathrm{~b}), B_{\text {sag,bld }}=0$ for $V_{l, t}>1 \%$ ). In addition, as shown in Figures $12(\mathrm{a})$, structure ST-SH was efficient in reducing the sagging distortions $\left(M^{D R, s a g}=0\right)$, whereas the $M^{D R, h o g}$ of this structure is greater than $M^{D R, h o g}$ of ST-H. Note that the greenfield $B_{h o g, g f}$ of ST-H and ST-SH are identical. The continuity of the structure across the greenfield inflection point clearly has a significant effect. In addition, the trends of $B_{\text {sag,bld }} / B_{h o g}, b l d$ and $M^{D R}$ against $V_{l, t}$ in Figures 11 and 12 are due to the variation in the shape of the greenfield settlement curves with volume loss. Finally, the increase of the sagging transverse length between ST-SH and ST-SSH resulted in a structural behaviour mode dominated by the sagging deformations rather than the hogging deflection (see Figure 12).

These results illustrate that a continuous simple beam does not respond to the sagging and hogging portions of the greenfield settlement trough as independent sub-structures; TSI mechanisms due to structural continuity of simple beams [i] resulted in differences between $B_{\text {sag,bld }} / B_{\text {hog,bld }}$ and $B_{\text {sag,gf }} / B_{\text {hog,gf }}$, [ii] affected modification factors $M^{D R}$, and [iii] differed for structures with primary sagging $(e / B=0-0.3)$ and primary hogging $(e / B>0.5)$ deformation modes.

\section{A MODIFICATION FACTOR BASED APPROACH}

In this section, the EL analysis method is adopted to study $M^{D R, s a g}, M^{D R, h o g}, M^{B, s a g}, M^{B, h o g}$, which are referred to as 'flexural interaction parameters'. The influence of the weight of the structure on these parameters is neglected.

In the EL solution, by neglecting the horizontal greenfield movements and assuming that the greenfield settlement trough is given by a standard Gaussian curve (for both greenfield movements in clays and sands), the flexural interaction parameters depend on $E_{s}, \nu_{s}, i, E I^{*}$, which have previously been defined, and $D_{u}$ and $D_{l}$, which are maximum and minimum absolute offsets of the structure edges from the tunnel centreline, respectively, as shown in Figure 3. Note that $D_{u}>0$ by definition, whereas $D_{l}<0$ and $D_{l}>0$ indicate that $e / B<0.5$ and $e / B>0.5$, respectively. If the influence of $\nu_{s}$ is neglected as 

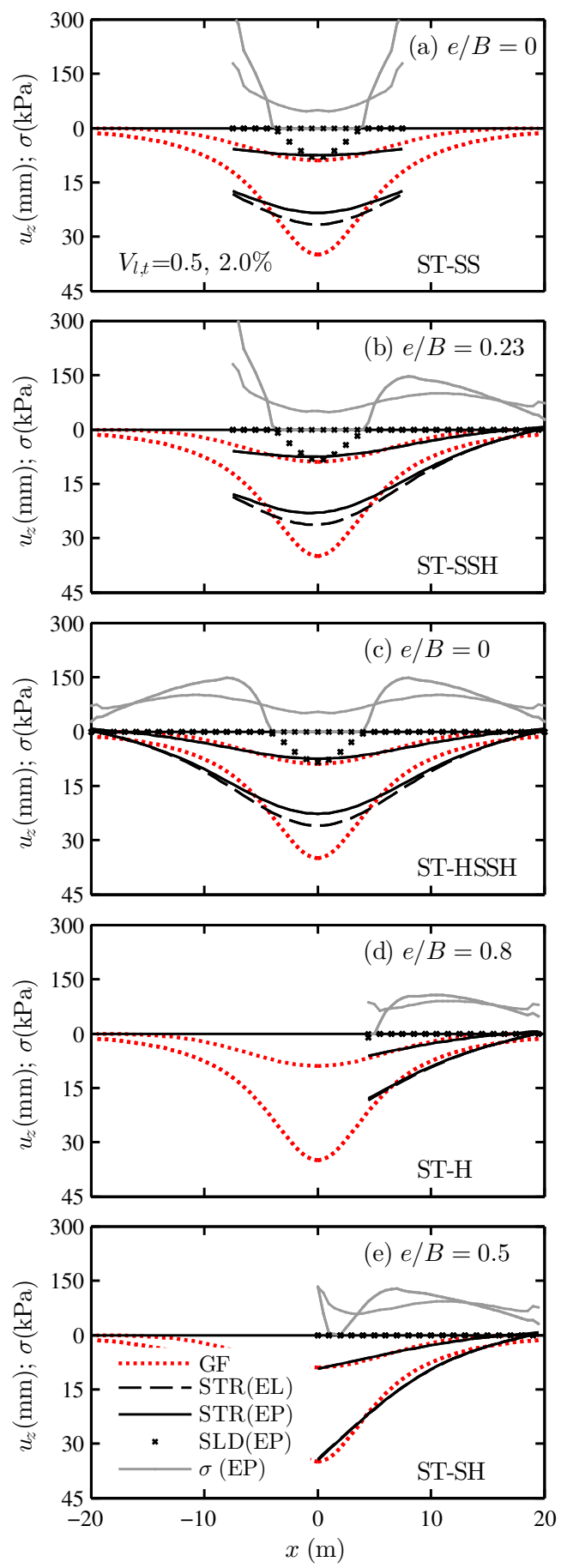

Fig. 10. Tunnelling-induced displacements and contact stress distributions: the effects of structure continuity.

$$
R=\frac{E I^{*}}{E_{s} i^{3}}, \quad \frac{D_{u}}{i}, \quad \frac{D_{l}}{i}
$$

where $R$ is the rigidity factor (Klar et al., 2005).

In this section, analyses were performed for varying stiffness values of the simple beam (see Table 4 ) and $L=1 \mathrm{~m}$. Assuming $L=1 \mathrm{~m}$ gives greater $M^{D R}$ compared to higher $L$ values or plane-strain conditions (Franzius et al., 2006). Additional analyses were performed to investigate the effects of $\nu_{s}$ on TSI. The sensitivity of flexural interaction parameters to Poisson's ratio was minor and $M^{D R}$ values associated with $\nu_{s}=0.25$ were lower than for $\nu_{s}=0.5$. In particular, the difference in $M^{D R}$ between $\nu_{s}=0.5$ and 0.25 was up to a value of 0.12 in a limited number of scenarios. This trend is in 

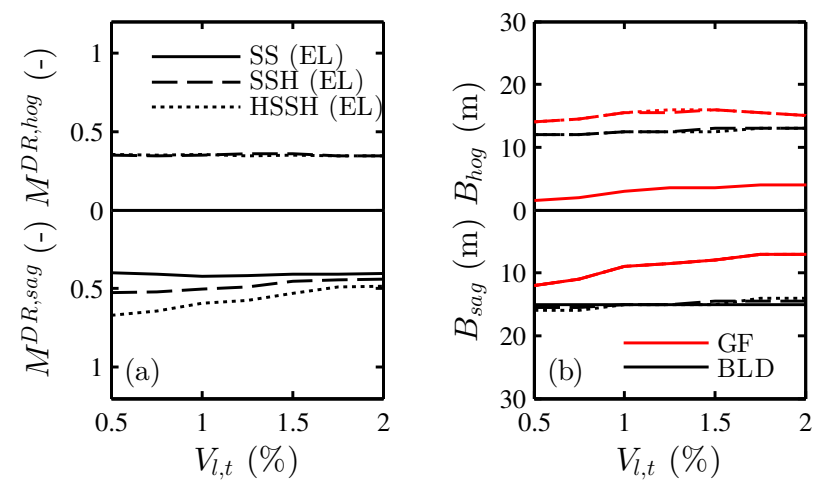

Fig. 11. Effects of the structure portion in the hogging zone on sagging deformations.
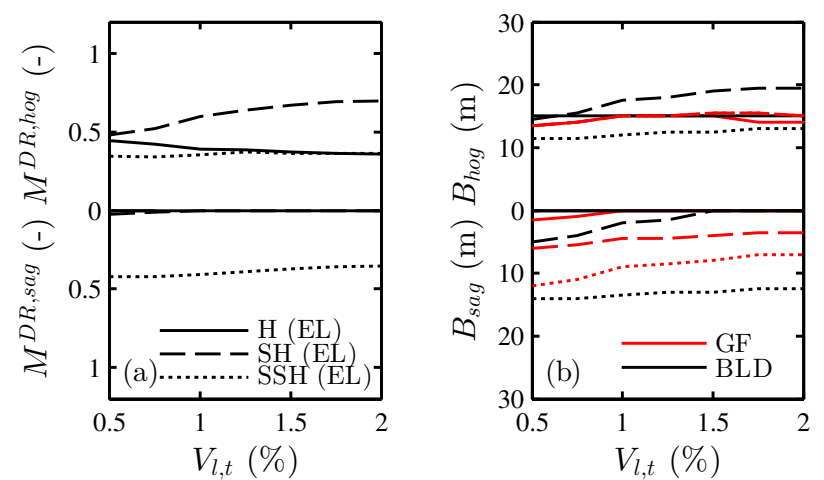

Fig. 12. Effects of the structure portion in the sagging zone on hogging deformations.

agreement with the fact that the subgrade modulus for an infinite beam on Winkler soil (Vesic, 1961) increases by $25 \%$ between $\nu_{s}=0.25$ and 0.5 ; a variation of soil stiffness by $25 \%$ in the semi-log scale of $M^{D R}-\rho$ charts has a minor impact. Thus, in the following, $\nu_{s}=0.5$ was assumed.

The following procedure was implemented to calculate the modification factors. Given the greenfield soil settlement profiles, the structure vertical displacement profile was calculated using the continuum solution proposed herein. Subsequently, the sagging and hogging zones of both the curves were defined as the regions extending between the inflection points (of the given curve) and/or the end of the structure; then, $M^{D R, s a g}, B_{s a g, g f}$, and $B_{\text {sag,bld }}$ were computed for the sagging zone above the tunnel, and $M^{D R, h o g}, B_{h o g, g f}$, and $B_{h o g, b l d}$ were computed for the sub-portion of the structure within the hogging zone with the maximum $D R^{h o g} / D R^{h o g, b l d}$. In other words, for hogging, a numerical procedure was used to calculate $D R_{\text {hog }} / D R_{\text {hog,bld }}$ for all possible sub-portions of the hogging zone, and the maximum value was selected.

This approach does not require the a priori definition of cut-off distances for the settlement curves (e.g. Mair et al. (1996) suggested to limit the structure transverse length to where the greenfield settlement is above $1 \mathrm{~mm}$ ). Also, note that for a standard Gaussian curve and an infinite structure, the maximum $D R^{\text {hog,gf }}$ occurs when the ends of the hogging portion of the structure are assumed to be at $x=i$ and $\approx 3.7 i$. This implies that, for long structures, the greenfield $B_{h o g, g f} \leq 2.7 i$.

Table 4. Bending stiffness for the parametric study.

\begin{tabular}{lll}
\hline Simple Beam -15 cases & $\min$ & $\max$ \\
\hline$E I^{*}\left(\mathrm{kNm}^{2} / \mathrm{m}\right)$ & $10^{2}$ & $10^{9}$ \\
\hline
\end{tabular}

Firstly, the relationship between $M^{D R}$ and both $\rho$ and $R$ are investigated for structures entirely located in greenfield sagging and hogging zones. Results are displayed in Figure 13 for greenfield $i=5 ; 10 \mathrm{~m}$, soil stiffness $E_{s}=8 ; 80 \mathrm{MPa}$, and structures in either pure sagging $\left(D_{l} / i=-1, D_{u} / i=1\right)$ or pure hogging $\left(D_{l} / i=1, D_{u} / i=2.5\right)$. Figure 13(a) shows agreement between the $M^{D R}-\rho$ data and the Mair (2013) envelopes, illustrating the suitability of the proposed EL analysis method to investigate the modification factors. Interestingly, both purely sagging and hogging structures are associated with the same $M^{D R}-\rho$ trend. A similar relationship between $M^{D R, s a g}$ and $\rho$ was achieved for purely sagging structures by Basmaji et al. (2017). Finally, results in Figure 13(b) confirm the appropriateness of the adopted dimensionless groups in Equation (15). 

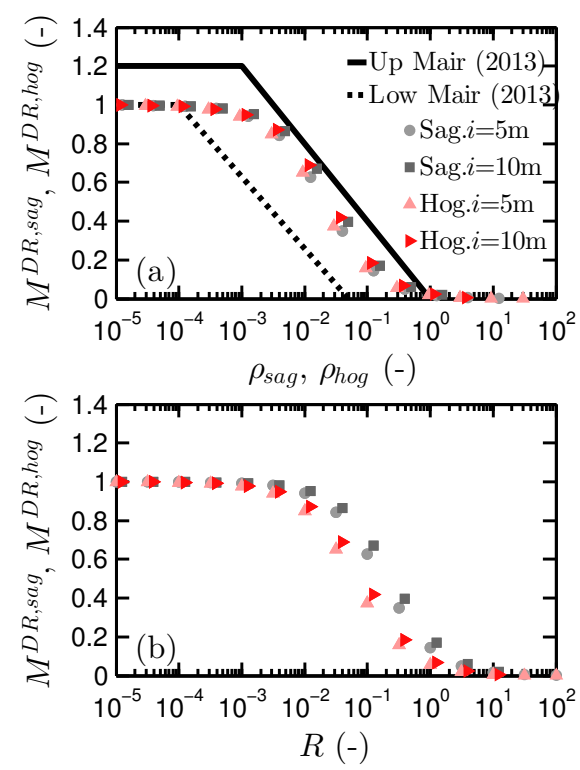

Fig. 13. $M^{D R}$ against (a) $\rho$ and (b) $R$ for structures entirely in sagging or hogging.
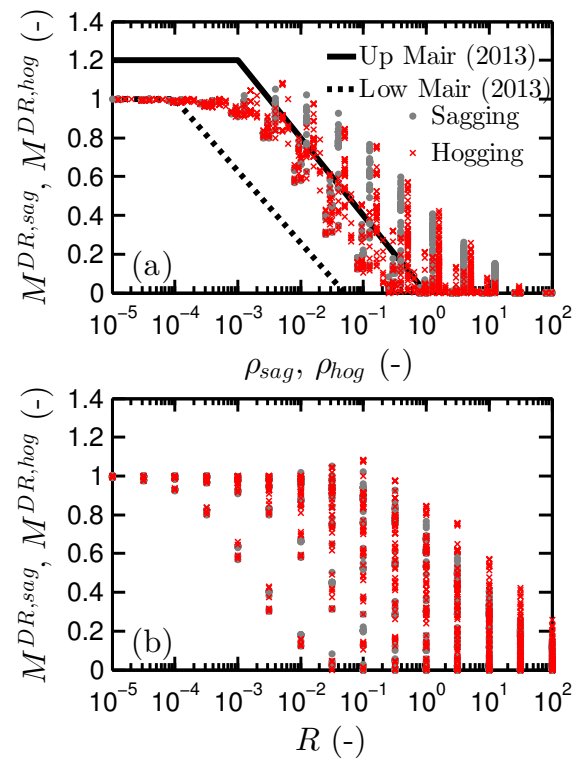

Fig. 14. Parametric study: $M^{D R}$ against (a) $\rho$ and (b) $R$.

Next, $M^{D R}$ of structures spanning both sagging and hogging zones is addressed with a parametric investigation for the intervals $D_{l} / i=\left[-D_{u} / i,+2\right]$ and $D_{u} / i=[0.5,10]$ (i.e. the entire range of possible structure locations) adopting $i=5 \mathrm{~m}$ and $E_{s}=80 \mathrm{MPa}$. This set of analyses is referred to as the 'parametric study' in the following.

For this parametric study, the trends in Figure 14(a) agree with the design curves of Mair (2013); however, there is a wide range of possible $M^{D R}$ corresponding to the interval $\rho=\left[10^{-2}, 10^{1}\right]$. Furthermore, Figure $14(\mathrm{~b})$ indicates that for a given rigidity factor $R$ the variability of $M^{D R}$ is also substantial.

Based on the concept that relative structure-soil stiffness could be defined with respect to the actual transverse length of the structure $B_{r}$ in the sagging and hogging zones (rather than for the greenfield lengths $B_{\text {sag,gf }}$ and $B_{h o g, g f}$ ), modified relative stiff factors $\rho_{\text {sag }}^{\prime}$ and $\rho_{h o g}^{\prime}$ are defined as

$$
\rho_{\text {sag } / \text { hog }}^{\prime}=\frac{E I^{*}}{E_{s} B_{\text {sag } / \text { hog }, \text { bld }}^{3}}=\frac{\rho_{\text {sag } / \text { hog }}}{\left(M^{B, \text { sag } / h o g}\right)^{3}}
$$

$M^{D R}$ against $\rho^{\prime}$ are plotted for the entire parametric study in Figure 15. By adopting $\rho^{\prime}$ rather than $\rho$, results are confined in a narrower region of the chart (compare Figures 14(a) and 15). Additionally, an excellent agreement is obtained between 
$M^{D R}-\rho^{\prime}$ in Figures $13(\mathrm{a})$ and 15 (for Figure $13(\mathrm{a}), M^{B, s a g}=M^{B, h o g}=1$ and $\rho_{\text {sag } / \text { hog }}=\rho_{\text {sag } / \text { hog }}^{\prime}$ ). Despite the efficiency of Equation (16), there is limited guidance for the estimation of $M^{B, s a g}$ and $M^{B, h o g}$, which is discussed in the following.

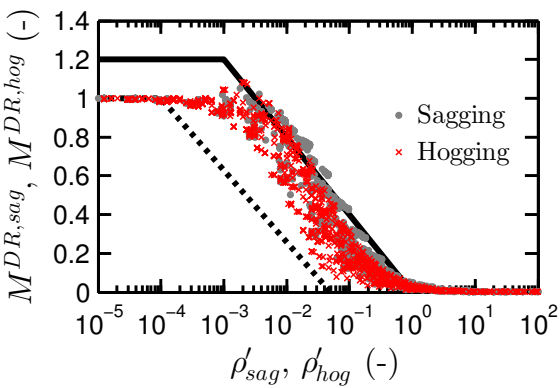

Fig. 15. Parametric study: $M^{D R}$ against $\rho^{\prime}$.

To distinguish between short and long structures either located in purely sagging/hogging or mixed zones, $M^{D R}$ and $M^{B}$ of the parametric study are plotted (lines) against $R$ in Figures 16 and 17 for all combination of $D_{u} / i=[1 ; 2 ; 3 ; 4 ; 7 ; 10]$ and $D_{l} / i=\left[-D_{u} / i ;-1 ;-0.5 ; 0 ; 0.5 ; 1\right]$. The parametric study results (lines) are plotted in the form of design charts. For validation purposes, they are displayed along with benchmark values (markers) derived from centrifuge tests, numerical simulation and field monitoring of tunnelling in clays and sands (Dimmock \& Mair, 2008; Farrell et al., 2014; Franzius et al., 2006; Haji et al., 2018; Lu et al., 2001; Mair \& Taylor, 2001; Potts \& Addenbrooke, 1997; Ritter et al., 2017b; Taylor \& Grant, 1998; Viggiani \& Standing, 2001). Benchmark data are coloured according to the closest available combination of $\left[D_{u} / i, D_{l} / i\right]$ in the plots. $\chi$ values were computed for centrifuge data based on the model structure and they were estimated for field case studies based on the available information. From each centrifuge test, several data points were defined for varying $V_{l, t}$. In particular, for a given tunnelling scenario in sand, the structure could be associated with different $D_{u} / i$ and $D_{l} / i$ values because of the decrease of $i$ with tunnel volume loss. In addition, soil stiffness degradation with tunnel volume loss (i.e. $E_{s}-V_{l, t}$ ) was considered for the datasets of Farrell et al. (2014) and Ritter et al. (2017b).

In Figure 16, there is a satisfactory agreement in terms of $M^{D R}$ between design curves and validation data for semiflexible structures in the range $R=\left[10^{-2}, 10^{2}\right]$, whereas there is an underestimation of the deformations of fully-flexible structures with $M^{D R}>1$ for $R<10^{-2}$. In particular, Figures 16 (a)-(f) display that, for a given $D_{u} / i$, the closer $D_{l} / i$ is to $D_{u} / i$, the lower $M^{D R, s a g}$. In addition, for a central structure $\left(e / B=0, D_{l} / i=-D_{u} / i\right.$, black solid lines $)$ there is a variation of $M^{D R, s a g}$ with $D_{u} / i$, whereas the influence of $D_{u} / i$ on $M^{D R, s a g}$ is limited for eccentric structures with $D_{l} / i=-1 ;-0.5 ; 0 ; 0.5$.

In Figures $16(\mathrm{~g})-(\mathrm{m}), M^{D R, h o g}$ curves shift towards greater rigidity factor $R$ with the increase in $D_{u} / i$, whereas the slope of the curve in the transition zone (where an increase in $R$ induced a decrease in $M^{D R, h o g}$ ) reduces with $D_{u} / i$. Regarding the influence of $D_{l} / i$ on $M^{D R, h o g}$, it is minor except for long structures with $e / B \approx 0.5\left(D_{u} / i=4-10\right.$ and $\left.D_{l} / i=0 ; 0.5\right)$, for which $M^{D R, h o g}$ tends to be slightly larger for $R>10^{-2}$ prior to the transition zone (see Figures 16(i)-(m)). This indicates that structures that are predominantly in the hogging region but extend up to halfway across the sagging region deform more than similar structures in pure hogging or that extend across the entire sagging region.

Next, the predictions of $M^{B, s a g}$ and $M^{B, h o g}$ are addressed. In Figures 17(a)-(f), $M^{B, s a g}$ increases and decreases with $R$ for central structures and eccentric structures, respectively; however, these trends of variation were highly dependent on $D_{u} / i$. In Figures $17(\mathrm{~g})-(\mathrm{m})$, for relatively short structures $\left(D_{u} / i \leq 4\right), M^{B, h o g}$ variation is limited for eccentric structures $\left(D_{l} / i=-0.5 ; 0 ; 0.5\right)$, whereas this modification factor decreases with $R$ for structures centrally located above the tunnel $\left(D_{l} / i=-D_{u} / i\right)$. For relatively long structures $\left(D_{u} / i \geq 7\right), M^{B, h o g}$ increases within $R=\left[10^{-2}, 10^{1}\right]$ for all structure geometries; then, for further increase in $R>10^{1}, M^{B, h o g}$ increase and decreases, respectively, in the case of eccentric and central structures. There is fair agreement between the EL solution predictions and benchmark data, except for Ritter et al. (2017a) centrifuge dataset of eccentric buildings (see Figure 17(d)). This difference between the EL solution and Ritter et al. (2017a) data can be due to the influence of the openings (resulting in the structure deflection due to shear deformations). In fact, $M^{B}$ may be estimated with curves in Figure 17 for structures deforming in bending; however, for structures with a pure shear deformation mode, Potts \& Addenbrooke (1997) analyses suggested that $M^{B}=1$ regardless of $E I$. Further studies are needed to address shear deformability effects on $M^{B}$.

The outcomes of the parametric study relating $M^{D R}$ and $M^{B}$ values to $R, D_{u} / i$, and $D_{l} / i$ are given in tabular form in the supplemental data. These tables can be used in engineering practice. To approximately consider the effects of weight, 

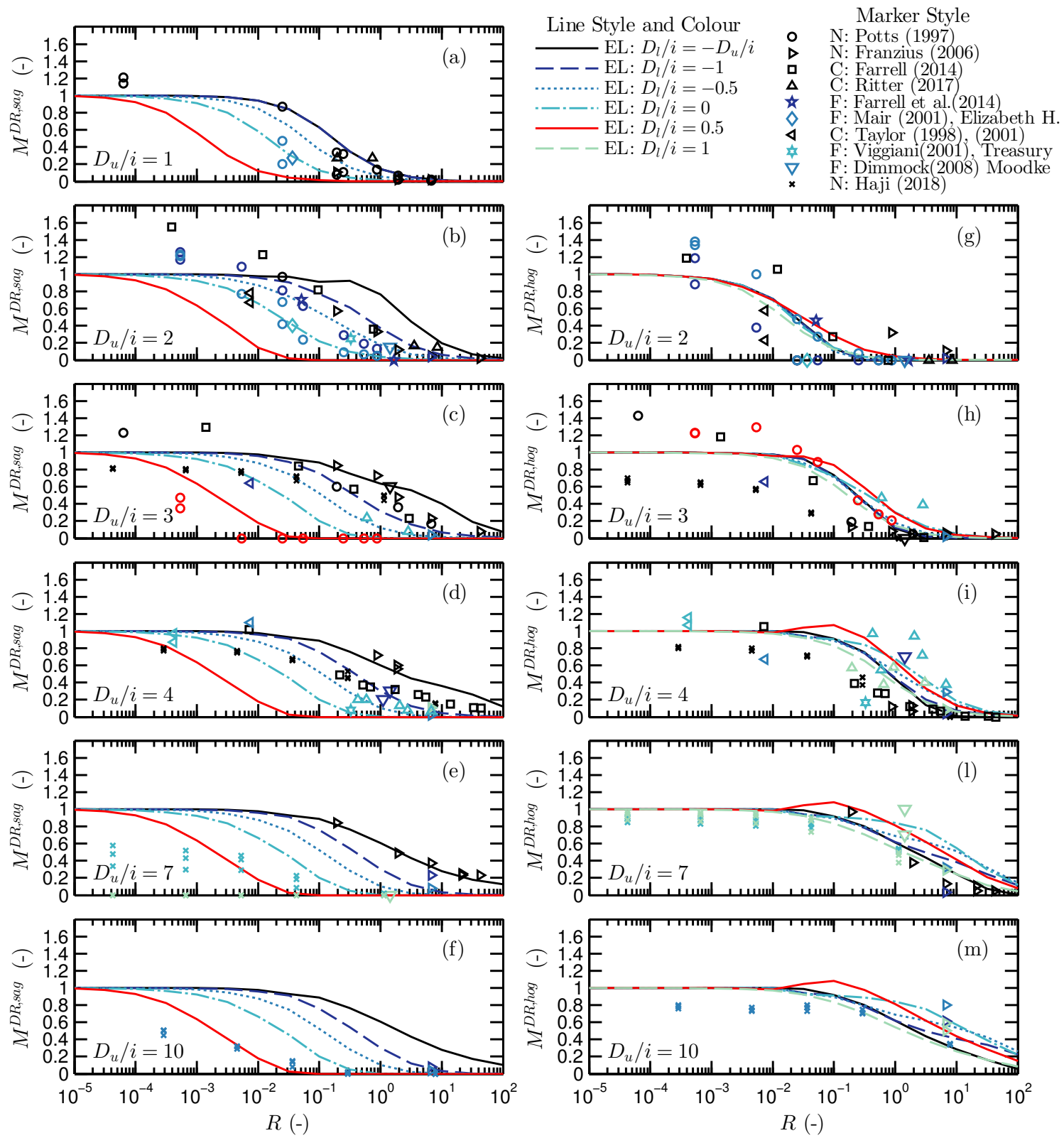

Fig. 16. Design curves of $M^{D R}$ against $R$ and comparison with centrifuge (C), numerical (N) and field (F) data.

for design purposes $M^{D R}$ could be estimated as follows

$$
M^{D R, \text { design }}=c^{W} M^{D R, c s} ; \quad c^{W}=1.2-1.4
$$

where $M^{D R \text {,design }}$ is the design value, and $M^{D R, c s}$ is obtained from the continuum solution. To summarise, the structure $D R$ and $B_{r}$ to be used in Equation (8) can be approximately estimated from the design charts for $M^{D R}$ and $M^{B}$ (see Figures 15, 16, and 17) while considering Equation (17) and greenfield movements.

\section{CONCLUSIONS}

Continuum solutions implementing equivalent simple beam model were used to predict tunnelling-induced deformations of structures. The following conclusions are drawn:

1. Equivalent stiffness $E I^{*}$ and $E A^{*}$ per meter run allowed accounting for openings as well as the foundation scheme through Melis \& Rodriguez Ortiz (2001) reduction term $(\alpha)$ and two newly defined factors $(\chi$ and $\lambda)$. 

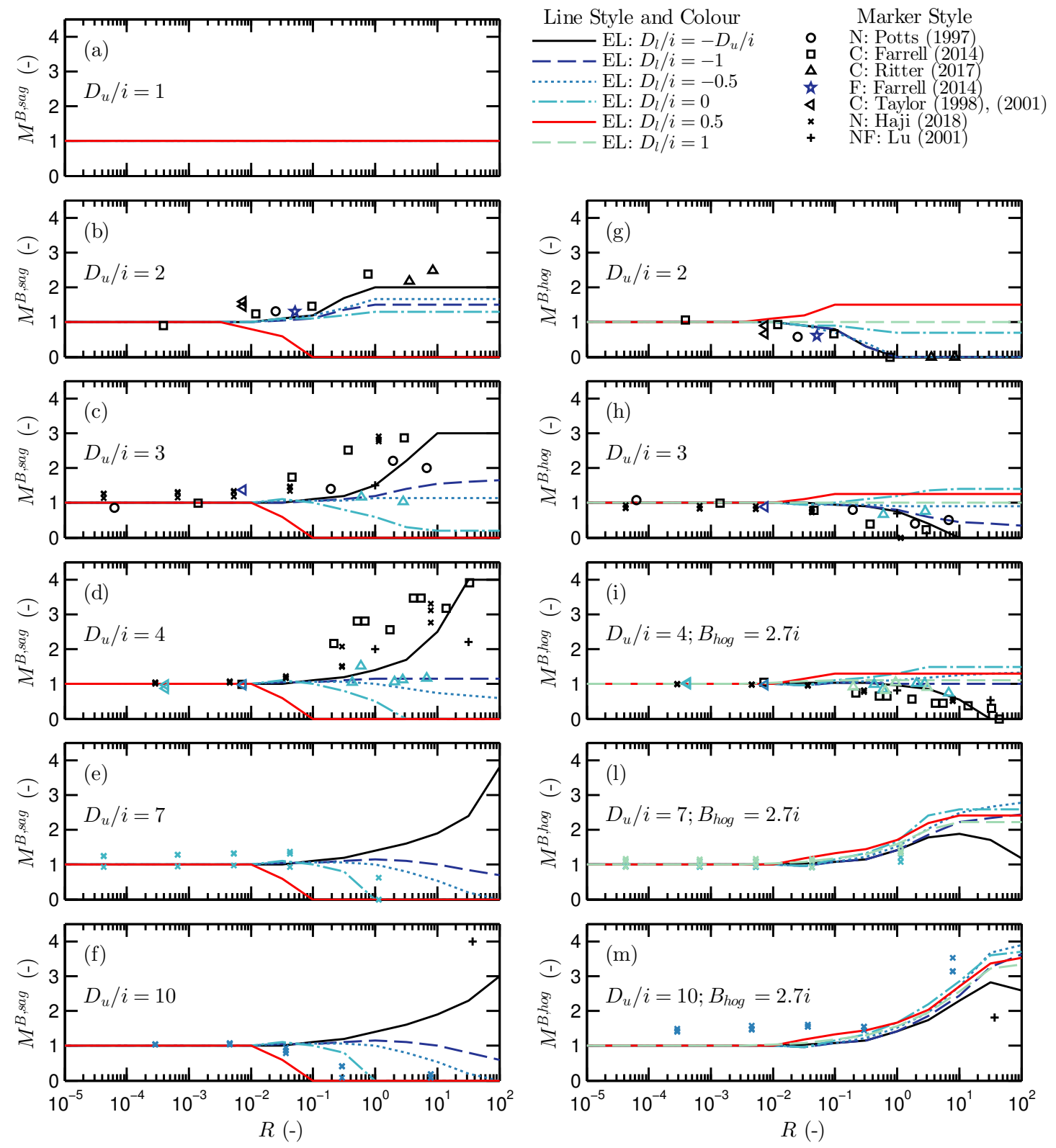

Fig. 17. Design curves of $M^{B}$ against $R$ and comparison with centrifuge (C), numerical (N) and field (F) data.

2. For structures spanning across greenfield sagging and hogging regions, the locations of the inflection points of the structure, $i_{b l d}$, and the greenfield settlement curve, $i$, differed because of the continuity of semi-flexible simple beams. This interaction mechanism varied for low and high values of tunnel-structure eccentricity. Further work is needed to assess the influence of the structural shear deformability.

3. For a given structure, flexural tunnel-structure interaction depends on the width of the greenfield settlement trough, which is related to $i$. A modified framework was proposed to assess modification factors of deflection ratios $\left(M^{D R}\right)$ as well as building transverse lengths $\left(M^{B}\right)$ in sagging/hogging zones. The design charts and the tabular results from the parametric analysis provide a means to implement the proposed framework in practice.

\section{ACKNOWLEDGEMENTS}

This work was supported by the Engineering and Physical Sciences Research Council (EPSRC) [EP/K018221/1, EP/N509620/1] and Crossrail. The authors are grateful to Dr. S. Acikgoz and Prof. R.J. Mair for valuable comments and to Dr R.P. Farrell for sharing experimental data. The associated research data and design charts are available at [link will be provided in the final version]. 
NOTATION

a

$b_{b, m}$

$d_{b, m}$

$c^{w}$

e

$g$

$i$

$i_{b l d}$

$i_{x}$

$n$

$q_{z, m}$

$t$

$u_{x}$

$u_{z}$

$u_{x, \max }$

$u_{z, \max }$

$x$

$z$

$z_{b}$

$z_{g}$

$z_{t}$

A

$A_{m}$

$A_{f}$

$A_{s}$

B

$B_{r}$

368

$B_{\text {sag } / \text { hog,bld }}$

$B_{\text {sag } / h o g, g f}$

C

D

$D_{l}$

$D_{u}$

$D R_{\text {sag } / \text { hog }, \text { bld }}$

$D R_{\text {sag } / \text { hog }, g f}$

E

$E_{m}$

$E_{s}$

$E A$

$E A^{*}$

EI

$E I^{*}$

G

H

I

$I_{m}$

$I_{d}$

L

$M^{B}$

$M^{D R}$

N

O

R

$V_{l, s}$

parameter of the modified Gaussian curve

equivalent beam cross-sectional width

equivalent beam cross-sectional depth

weight coefficient

building eccentricity

gravity

distance between the centreline and the greenfield inflection point

distance between the centreline and the inflection point of the building settlement trough

distance between the centreline and maximum horizontal displacement

shape factor used in modified Gaussian curve

simple beam load

distance between the neutral axis and the edge of the beam in tension

horizontal movement

vertical movement

maximum horizontal displacement

maximum vertical displacement

horizontal offset distance from tunnel centreline

depth, measured from ground surface

neutral axis level for pure bending deformations

ground level

depth of tunnel axis

cross-sectional area

cross-sectional area of the equivalent beam model

contact area of the foundation

contact area of the building

building transverse length

building length in the sagging or hogging region

transverse length of the sagging/hogging region of the building settlement trough

transverse length of the sagging/hogging region of the greenfield settlement trough

cover: distance from surface to tunnel crown

tunnel diameter

minimum offset of the structure edges from the tunnel centreline

maximum offset of the structure edges from the tunnel centreline

deflection ratio in the sagging/hogging region of the building settlement trough

deflection ratio in the sagging/hogging region of the greenfield settlement trough

Young's modulus of the structure

Young's modulus of the equivalent beam model

Young's modulus of the soil

axial stiffness

axial stiffness per $m$ run

bending stiffness

bending stiffness per $m$ run

shear modulus

building height

second moment of area

second moment of area of the equivalent beam model

relative density

building longitudianl length

modification factor for the sagging and hogging region length

modification factor for the deflection ratio

centrifuge acceleration

ratio of façade openings

rigidity factor

volume loss of soil

PrKpared using GeotechAltkmecs loss of tunnel 
EL elastic solution

EP elastoplastic solution

EXP experimental

SLD slider

STR structure/building

$\alpha \quad$ bending stiffness reduction factor

$\chi \quad$ ratio between foundation and building areas

\section{REFERENCES}

Basmaji, B., Deck, O. \& Al Heib, M. (2017). Analytical model to predict building deflections induced by ground movements. European Journal of Environmental and Civil Engineering , 1-23doi:10.1080/19648189.2017.1282382.

Bilotta, E., Paolillo, A., Russo, G. \& Aversa, S. (2017). Displacements induced by tunnelling under a historical building. Tunnelling and Underground Space Technology 61, 221-232, doi:10.1016/j.tust.2016.10.007.

Boscardin, M. D. \& Cording, E. J. (1989). Building response to excavation-induced settlement. Journal of Geotechnical Engineering 115, No. 1, 1-21.

Burd, H. J., Houlsby, G. T., Augarde, C. E. \& Liu, G. (2000). Modelling tunnelling-induced settlement of masonry buildings. Proceedings of the Institution of Civil Engineers-Geotechnical Engineering 143, No. 1, 17-29.

Burland, J. B., Mair, R. J. \& Standing, J. R. (2004). Ground performance and building response due to tunnelling. In Advances in Geotechnical Engineering: The Skempton Conference - Proceedings of a Three Day Conference on Advances in Geotechnical Engineering, organised by the Institution of Civil Engineers, vol. 1 (Jardine, R. J., Potts, D. M. \& Higgins, K. G., eds.), London, United kingdom: Thomas Telford Services Ltd, pp. 291-344.

Burland, J. B. \& Wroth, C. P. (1974). Settlement of buildings and associated damage. In Proceedings of the conference on settlement of structures, London, UK: Pentech Press, pp. 611-654.

Dimmock, P. S. \& Mair, R. J. (2008). Effect of building stiffness on tunnelling-induced ground movement. Tunnelling and Underground Space Technology 23, No. 4, 438-450, doi:10.1016/j.tust.2007.08.001.

Fargnoli, V., Gragnano, C. G., Boldini, D. \& Amorosi, A. (2015). 3D numerical modelling of soilâĂŞstructure interaction during EPB tunnelling. Géotechnique 65, No. 1, 23-37, doi:10.1680/geot.14.P.091.

Farrell, R. (2010). Tunnelling in sands and the response of buildings. Ph.D. Thesis, Cambridge University .

Farrell, R., Mair, R., Sciotti, A. \& Pigorini, A. (2014). Building response to tunnelling. Soils and Foundations 54, No. 3, 269-279, doi:10.1016/j.sandf.2014.04.003.

Franza, A. \& DeJong, M. J. (2018). Elastoplastic solutions to predict tunnelling-induced load transfer and deformation mechanisms of surface structures. Journal of Geotechnical and Geoenvironmental Engineering (In Press) doi:10.1061/(ASCE)GT.1943-5606. 0002021.

Franza, A. \& Marshall, A. M. (2018). Centrifuge Modeling Study of the Response of Piled Structures to Tunneling. Journal of Geotechnical and Geoenvironmental Engineering 144, No. 2, 04017109, doi:10.1061/(ASCE)GT.1943-5606.0001751.

Franza, A., Marshall, A. M., Haji, T., Abdelatif, A. O., Carbonari, S. \& Morici, M. (2017). A simplified elastic analysis of tunnel-piled structure interaction. Tunnelling and Underground Space Technology 61, No. January, 104-121, doi:10.1016/j.tust.2016.09.008.

Franza, A., Marshall, A. M. \& Zhou, B. (2018). Greenfield tunnelling in sands: the effects of soil density and relative depth. Géotechnique (In press) doi:10.1680/jgeot.17.p.091.

Franzius, J. N., Potts, D. M., Addenbrooke, T. I. \& Burland, J. B. (2004). The influence of building weight on tunnelling-induced ground and building deformation. Soils and Foundations 44, No. 1, 25-38.

Franzius, J. N., Potts, D. M. \& Burland, J. B. (2006). The response of surface structures to tunnel construction. Proceedings of the ICE - Geotechnical Engineering 159, No. 1, 3-17, doi:10.1680/geng.2006.159.1.3.

Frischmann, W., Hellings, J., Gittoes, G. \& Snowden, C. (1994). Protection of the Mansion House against damage caused by ground movements due to the Docklands Light Railway Extension. Proceedings of the Institution of Civil Engineers - Geotechnical Engineering 107, No. 2, 65-76, doi:10.1680/igeng.1994.26374. 
Giardina, G., DeJong, M. J. \& Mair, R. J. (2015). Interaction between surface structures and tunnelling in sand: Centrifuge and computational modelling. Tunnelling and Underground Space Technology 50, 465-478, doi:10.1016/j.tust.2015.07.016.

Haji, T. K., Marshall, A. M. \& Franza, A. (2018). Mixed empirical-numerical method for investigating tunnelling effects on structures. Tunnelling and Underground Space Technology 73, 92-104, doi:10.1016/j.tust.2017.12.008.

Klar, A., Vorster, T. E. B., Soga, K. \& Mair, R. J. (2005). Soil-pipe interaction due to tunnelling : comparison between Winkler and elastic continuum solutions. Géotechnique 55, No. 6, 461-466.

Losacco, N., Burghignoli, A. \& Callisto, L. (2014). Uncoupled evaluation of the structural damage induced by tunnelling. Géotechnique 64, No. 8, 646-656, doi:10.1680/geot.13.P.213.

Lu, Y.-C., Bloodworth, A. G. \& Gleig, F. D. (2001). Behaviour of long structures in response to tunnelling. In Proceedings of the international conference Response of buildings to excavation-induced ground movements (Jardine, F. M., ed.), London, UK: CIRIA, pp. 367-374.

Mair, R. (2013). Tunnelling and deep excavations: ground movements and their effects. In Proceedings of the 15th European Conference on Soil Mechanics and Geotechnical Engineering - Geotechnics of Hard Soils - Weak Rocks (Part 4) (Anagnostopoulos, A., Pachakis, M. \& Tsatsanifos, C., eds.), Amsterdam, the Netherlands: IOS Press, pp. 39 - 70.

Mair, R. J. \& Taylor, R. N. (2001). Elizabeth House: settlement predictions. In Building response to tunnelling: case studies from the Jubilee Line Extension, London. Volume 1: Projects and Methods (Burland, J. B., Standing, J. R. \& Jardine, F. M., eds.), London, United Kingdom: CIRIA and Thomas Telford, pp. 195-215.

Mair, R. J., Taylor, R. N. \& Bracegirdle, A. (1993). Subsurface settlement profiles above tunnels in clay. Géotechnique 43, No. 2, 315-320, doi:10.1680/geot.1993.43.2.315.

Mair, R. J., Taylor, R. N. \& Burland, J. B. (1996). Prediction of ground movements and assessment of risk of building damage due to bored tunnelling. In Proceedings of the International Symposium on Geotechnical Aspects of Underground Construction in Soft Ground (Mair, R. J. \& Taylor, R. N., eds.), London, United Kingdom: Balkema, Rotterdam, pp. 713-718.

Marshall, A. M., Farrell, R., Klar, A. \& Mair, R. (2012). Tunnels in sands: the effect of size, depth and volume loss on greenfield displacements. Géotechnique 62, No. 5, 385-399, doi:10.1680/geot.10.P.047.

Melis, M. J. \& Rodriguez Ortiz, J. M. (2001). Consideration of the stiffness of buildings in the estimation of subsidence damage by EPB tunnelling in the Madrid subway. In Proceedings of international conference: response of buildings to excavation induced ground movements, London, London, UK: Ciria Special Publication 201, pp. 87-394.

Namazi, E. \& Mohamad, H. (2013a). Assessment of Building Damage Induced by Three-Dimensional Ground Movements. Journal of Geotechnical and Geoenvironmental Engineering 139, No. 4, 608-618, doi:10.1061/(ASCE)GT.1943-5606.0000822.

Namazi, E. \& Mohamad, H. (2013b). Potential damage assessment in buildings undergoing tilt. Proceedings of the Institution of Civil Engineers-Geotechnical Engineering 166, No. 4, 365-375, doi:10.1680/geng.10.00132.

Peck, R. B. (1969). Deep excavations and tunnelling in soft ground. In Proceedings of the 7th International Conference on Soil Mechanics and Foundation Engineering, Mexico City, Mexico, pp. 225-290.

Pickhaver, J., Burd, H. \& Houlsby, G. (2010). An equivalent beam method to model masonry buildings in 3D finite element analysis. Computers \& Structures 88, No. 19, 1049-1063, doi:10.1016/j.compstruc.2010.05.006.

Potts, D. M. \& Addenbrooke, T. I. (1997). A structure's influence on tunnelling-induced ground movements. Proceedings of the ICE - Geotechnical Engineering 125, No. 2, 109-125.

Ritter, S. (2017). Experiments in tunnel-soil-structure interaction. Ph.D. thesis, University of Cambridge doi:10.17863/CAM.20966.

Ritter, S., Giardina, G., DeJong, M. J. \& Mair, R. J. (2017a). Centrifuge modelling of building response to tunnel excavation. International Journal of Physical Modelling in Geotechnics doi:10.1680/jphmg.16.00053.

Ritter, S., Giardina, G., DeJong, M. J. \& Mair, R. J. (2017b). Influence of building characteristics on tunnelling-induced ground movements. Géotechnique 67, No. 10, 926-937, doi:10.1680/jgeot.SIP17.P.138.

Sugiyama, T., Hagiwara, T., Nomoto, T., Nomoto, M., Ano, Y., Mair, R., Bolton, M. \& Soga, K. (1999). Observations of ground movements during tunnel construction by slurry shield method at the Docklands Light Railway Lewisham Extension-East London. Soils and Foundations 39, No. 3, 99-112.

Taylor, R. N. \& Grant, R. J. (1998). Centrifuge modelling of the influence of surface structures on tunnelling induced ground movements. In Tunnels and Metropolises, pp. 261-266.

Taylor, R. N. \& Yip, D. L.-F. (2001). Centrifuge modelling of the effect of a structure on tunnel-induced ground movements. In Proceedings of the International Conference on the response of buildings to excavation-induced ground movements (Jardine, F. M., ed.), London, UK: CIRIA, pp. 601-611.

Vaziri, H., Simpson, B., Pappin, J. W. \& Simpson, L. (1982). Integrated forms of Mindlin's equations. Géotechnique 32, No. 3, 275-278.

Vesic, A. B. (1961). Bending of beams resting on isotropic elastic solid. Journal of the Engineering Mechanics Division 87, No. EM2, Part 1, 35-53.

Viggiani, G. \& Standing, J. (2001). The Treasury. In Building response to tunnelling: case studies from the Jubilee Line Extension, London. Volume 2: Case studies (Burland, J. B., Standing, J. R. \& Jardine, F. M., eds.), CIRIA and Thomas Telford, pp. $401-423$.

Vorster, T. E. B., Klar, A., Soga, K. \& Mair, R. J. (2005). Estimating the Effects of Tunneling on Existing Pipelines. Journal of Geotechnical and Geoenviromental Engineering 131, No. 11, 1399-1410, doi:10.1061/(ASCE)1090-0241(2005)131:11(1399). 